\title{
Towards 3D printed saxophone mouthpiece personalization: Acoustical analysis of design variations
}

\author{
Mehmet Ozdemir ${ }^{1,3,{ }^{*} \text { (D), Vasileios Chatziioannou }}{ }^{2}$ (D), Jouke Verlinden ${ }^{3}$ (D), Gaetano Cascini $^{1}$ (D), \\ and Montserrat Pàmies-Vilà ${ }^{2}$ (i) \\ ${ }^{1}$ Department of Mechanical Engineering, Politecnico di Milano, 20156 Milan, Italy \\ ${ }^{2}$ Department of Music Acoustics - Wiener Klangstil, University of Music and Performing Arts Vienna, 1030 Vienna, Austria \\ ${ }^{3}$ Department of Product Development, University of Antwerp, 2000 Antwerp, Belgium
}

Received 15 March 2021, Accepted 22 September 2021

\begin{abstract}
Saxophonists have different expectations from the saxophone mouthpiece, as it significantly affects the playability and the sound of the instrument. A mass personalization paradigm provides unique products to cater to their needs, using the flexibility of additive manufacturing. The lack of quantitative knowledge on mouthpiece design hinders the personalization attempts. This study aims to lay out how design parameters affect mouthpiece characteristics. Twenty-seven 3D-printed mouthpieces with varying design parameters are used in conjunction with an artificial blowing machine, to determine the acoustical relevance of the various mouthpiece designs on four selected mouthpiece features. The influence of the design parameters is evaluated statistically and via a case study with five saxophonists. The analysis shows that seven out of nine parameters tested affect the mouthpiece characteristics by relatively different amounts. A user study demonstrates that saxophonists confirm the results in 7 of 10 cases, and they prefer personalized mouthpieces in 4 of 5 cases. The results present a key contribution to the understanding of mouthpiece design. The findings provide valuable insights for new mouthpiece design and mouthpiece personalization.
\end{abstract}

Keywords: Single-reed woodwinds, Mass personalization, 3D printing, Mouthpiece design, Artificial blowing

\section{Introduction}

The possibility to characterize musical instruments, or parts thereof, prior to manufacture, is an invaluable asset for instrument makers. Similarly, after manufacture, it is beneficial to be able to assess some properties of an instrument in the absence of the player. The former approach is based on virtual designs of the instrument, whereas the latter requires an objective characterization, while exciting the instrument by artificial means. This study combines the above approaches in order to investigate design personalization for saxophone mouthpieces.

The saxophone sound is produced by the oscillation of a single reed as the air flows through the internal cavity of the mouthpiece [1]. The volume and the internal geometry of the mouthpiece have been shown to affect the input impedance of the instrument and hence the resulting sound [2, 3]. Moreover, small design changes on the mouthpiece may significantly affect the oscillations of the reed and cause playability, intonation or timbre differences [3-5]. For instance, wider tip openings result in higher thresholds of oscillation $[6,7]$, requiring higher blowing pressure to start a tone.

*Corresponding author: mehmet.ozdemir@polimi.it
Furthermore, it has been shown via both experimental measurements [8] and physical modeling [9, 10] that reed stiffness dynamically increases when the reed closes, hence the reed-mouthpiece interface might affect the oscillation threshold and other playing parameters. Introducing changes in the mouthpiece geometry, though, might lead to either meeting the players' individual desires or generating counterproductive results (e.g., affecting the tuning of the instrument).

The mouthpiece is a very personal product, both in terms of playability and produced sound. Therefore, players have different expectations and desires when choosing a mouthpiece. To be able to provide the desired features in the mouthpiece, the effects of the design changes need to be determined. This requires an understanding of the results of the design changes precisely. The traditional approach taken by manufacturers and artisans to improve or tailor the mouthpiece features is based on trial-and-error and iterating the changes in the design aspects [11]. Mouthpieces today exhibit a wide range of designs, some being quite different from the original design by Adolphe Sax, as the manufacturers iterated design changes based on players' feedback over the years [12]. These mouthpieces are sometimes further modified or fine-tuned by artisans 
to meet the players' demands. However, given that design aspects and their changes have no standard, the evaluation of these changes is very subjective, and it is challenging to tailor the mouthpiece to the desired performance. Besides, the lack of objective measures results in misinformation about the mouthpiece design, such as the common false belief of the mouthpiece material's effect on sound [4].

This work is an application of a design personalization methodology to a specific case considering saxophone mouthpieces, which is particularly relevant to the field of music acoustics. The overarching goal is to mass-personalize saxophone mouthpieces according to the mass personalization model suggested in previous work [13]. This model is the result of a methodical process in which mouthpiece design parameters are changed to meet different performance needs of players. To achieve the relevant mouthpiece information for this process, the present study aims to quantify the effect of mouthpiece design parameters (hereafter parameters) on the playability and the sound of the instrument (hereafter features). Playability and sound criteria are defined by objective indicators extracted from the measurements obtained with an artificial blowing machine and one musician. These tests consider a set of 27 different mouthpiece designs configured according to the Taguchi method [14]. Analysis of the experiment results provides the quantitative relationships between mouthpiece features and design parameters (Sect. 5). These quantitative relationships are then used to build a mass personalization model that estimates the geometry of a mouthpiece considering the given player needs. Subjective evaluation of the personalization model is presented at the end of this manuscript in a userstudy with five saxophonists.

The layout of the paper is as follows; Section 2 provides background information on mouthpiece design and personalization; Section 3 explains the methodology of the study; Section 4 presents the processing of the experimental data and the statistical analysis of the results; Section 5 gives the discussion and interpretation of the results; Section 6 presents the personalization model and the user study; Sections 7 and 8 present the general discussion and the conclusion.

\section{State of the art}

\subsection{Mouthpiece design}

Saxophone mouthpiece design has transformed since the invention of the saxophone, and several iterations from the original design exist today [12]. These changes in the mouthpiece design have been proven to provide distinct tonal characteristics $[5,15]$. The key design aspects in mouthpiece structure are shown in Figure 1.

Both the description and perception of tone quality are subjective. Hence, it is difficult to describe the tonal characteristics and standardize them. There is an effort in psychoacoustics to relate the perceptual descriptions of saxophone sound to acoustical dimensions [16]. The formant-like structures in the frequency spectrum of the saxophone sound are considered to be defining its tonal characteristics [17], such as, e.g., "brightness", which may be assessed using the spectral centroid [18].

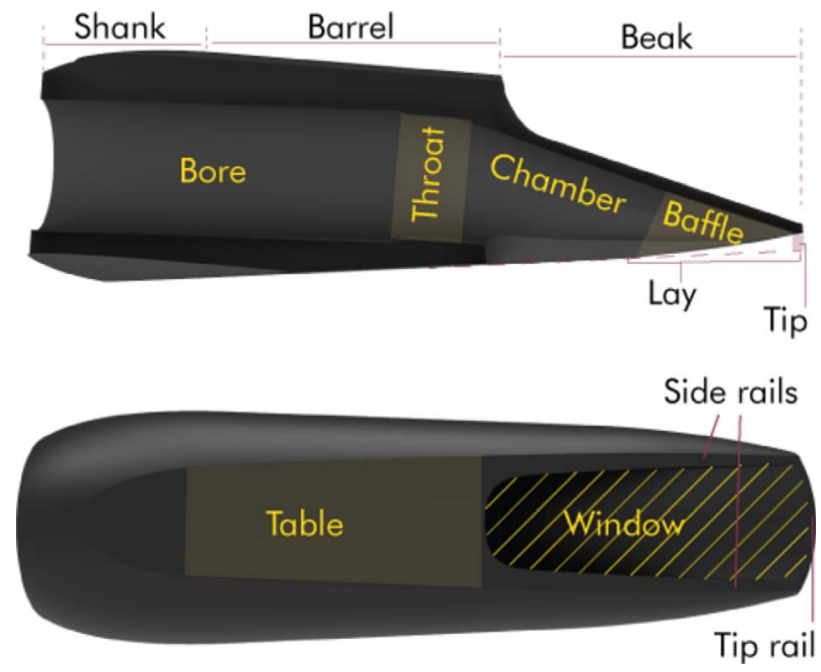

Figure 1. Saxophone mouthpiece design aspects.

Other than the tone quality, there are also performancerelated aspects of the mouthpiece that rely on its design, such as pitch, loudness, equality of registers, flexibility and ease of playing [4]. A steady pitch and good intonation throughout all registers are usually standard expectations from a mouthpiece. However, expectations regarding loudness, flexibility and ease of playing aspects are likely to differ, because of playing habits, music genre or playing environment [5].

The shape of the internal cavity (throat and chamber for the saxophone) is found to change the tone color and harmonic spectra of woodwind instruments [19]. On top of the change in tone quality, Teal [4] describes how mouthpiece dimensions and shape affect also loudness, flexibility and ease of playing. He further lists the effective parameters as tip opening, lay length, baffle height, tip rail width, chamber size and shape. Similarly, Pinksterboer [20] identifies tip opening, lay length, baffle shape and chamber size as important parameters affecting sound and playability. Baffle height is particularly associated with brightness or darkness of the tone [21]. In addition, variation in tip opening is found to alter control, loudness and projection of the mouthpiece. Analyzing and iterating the original design by Adolphe Sax, Celentano [12] identified chamber size, tip opening, table size and window size as the important parameters for playability and timbre. By examining the characteristics of the air flow through the mouthpiece, Lorenzoni demonstrated the effect of the inner geometry on the air flow, and hence on the produced sound [22].

The majority of the commercially available saxophone mouthpieces are manufactured with conventional methods. Therefore, tailoring the design for each player becomes challenging. At this point, additive manufacturing (AM) provides the necessary flexibility, as it eliminates the need of dedicated tooling to each design. The applications of $\mathrm{AM}$ for various wind instruments has already been demonstrated for various purposes, including restoration of ancient instruments, personalization or innovation [23, 24]. An example of an innovation case is shown by Hang, 3D 
printing a single-component mouthpiece with a built-in reed [25]. 3D printed custom mouthpieces are also available commercially [26]. Recreation of the original mouthpiece design by Adolphe Sax is also an excellent demonstration of the restoration value of AM [12]. As an attempt to alter the properties of the mouthpiece, Lorenzoni proposed novel designs of baffle and throat, which would have been very challenging with traditional manufacturing methods [27].

\subsection{Design personalization}

Perception of tone quality is very subjective, and it is likely to be defined by different adjectives by listeners [28]. The same subjectivity applies to producing sound with the mouthpiece and its associated playability, since the vocal tract of the player has an essential role in these [29-31]. Therefore, the same mouthpiece cannot guarantee the same sound when used by different players. Besides, conditions such as different music styles (e.g., classical or jazz) or playing environment may require different performance from the mouthpiece [20]. As a result, players have different needs and expectations, which makes the mouthpiece a very personal product.

The design personalization of the saxophone mouthpiece is handled in the perspective of mass personalization (MP) in this study. In MP paradigms, the aim is to provide unique products catering the individual needs of customers while sustaining near mass production efficiency [32]. The design process actively involves the customer to meet personal needs, while the flexibility of digital manufacturing (i.e., 3D printing) and design automation provides the efficiency. An application of MP on saxophone mouthpiece design is presented in previous work [13], where it is demonstrated that, in order to achieve MP for the mouthpiece, its personalizing features are to be identified, and to be expressed quantitatively in terms of design parameters. Since previous literature indicates a tangled relationship between the features and the parameters, the Taguchi method [14] of design of experiments (DOE) was used to devise an experiment. The following section explains the methodology of the experiment.

\section{Methodology}

The approach taken in the present work, in essence, is similar to the traditional empirical method of artisans, but in a constructed way based on quantitative design iterations. To reduce subjectivity in the design changes, both the design features and their interval of changes need to be defined parametrically. It is necessary to test the design parameters at different levels and analyze the variations they result in, to understand their behavior. Firstly, the mouthpiece features to examine were selected, and following that, corresponding design parameters were identified. A commercial alto saxophone mouthpiece was used as a base design, and a design template was created where parameters can be given a value within certain intervals. To understand the results of variations in design parameters, an experiment was devised to test mouthpieces with different design configurations, using an artificial blowing machine.

\subsection{Selected mouthpiece features}

Features of the mouthpiece may be grouped into two categories: timbre attributes and playability aspects. Especially in terms of defining the saxophone sound, or in general describing timbre, there are several descriptors identified in psychoacoustics [17]. However, these are based on the sound perception of the listener (player or external listener), hence very subjective. As the experiment requires quantitative measurements, the selection of the features to be tested with varying parameters is made by what is possible to be measured at the instrument. One of the most distinctive descriptors of timbre is brightness, which is related to the energy at the high-frequency partials in the spectrum. For this study, brightness is the only timbre attribute to be observed in the experiment. The playability aspects are somewhat more tangible since they appear at the player-mouthpiece interaction. Some of the significant aspects for players are blowing resistance, flexibility on the instrument's pitch, and the loudness of the produced sound. Therefore, the mouthpiece features to be considered in this work, their explanations, and means to be observed are as below:

- Resistance implies the difficulty of blowing into the mouthpiece, or in other terms, the ease of producing sound. For a fixed tip opening, the resistance of the mouthpiece is also dependent on the strength of the reed used. Therefore, the same synthetic reed is used to compare mouthpieces in this experiment, which is also independent of humidity changes. Resistance is assessed by considering the oscillation threshold [6], i.e., the blowing pressure necessary to start a sound.

- Loudness refers to the sound level of the instrument. Different loudness levels may be a desirable property for players. To exemplify, playing outdoors or in a marching band may require louder sounds, while the opposite may be valid for playing indoors. During performance, loudness is controlled by the players' blowing pressure to achieve different dynamics [33]. Yet being a perceptual feature, in order to define a standard to compare the tested mouthpieces, loudness is assessed in this work by considering the external sound pressure level at a fixed blowing air pressure.

- Brightness is the most common tone color descriptor among players to describe how bright or dark is the sound produced. As different levels of brightness might be a personal preference, it may be more suitable or desirable for different music genres. Brightness is associated with the high-frequency content in the spectrum of a sound and can be assessed via its spectral centroid [18].

- Flexibility, also referred to as pitch flexibility [34] or flexibility of intonation [35], describes the possibility to adjust pitch. Saxophonists can alter the pitch of a 
fingered note in order to vary its intonation or perform pitch modifications such as pitch bending or glissando [31]. Higher flexibility might be a desirable property for a jazz player, while for a classical player, lower flexibility, and more sustainable pitch might be of more value.

\subsection{Design parameters}

The design aspects used in the literature [4, 12, 20, 21] to produce saxophone mouthpieces (Fig. 1) can be grouped as reed interface (table, lay, tip opening, tip rail, side rails), internal airflow (bore, throat, chamber, baffle), and player interface (beak) aspects. The design parameters used in this work are derived from the definitions in the literature, focusing on the reed interface and internal airflow aspects. Player interface aspects are disregarded because they would not result in an observable effect when tested in an artificial blowing setup. For instance, beak size does not directly affect the airflow or reed oscillation; however, it might affect the player's oral cavity and hence indirectly the airflow [4]. Furthermore, changes in tip and side rail thicknesses are significant when there is lip pressure change or tonguing exercise. However, since neither are present in the experiments, these parameters are excluded as well. One last reason to exclude a design parameter is the geometrical dependence between parameters: facing curve radius, lay length, and tip opening are dependent variables (Fig. 2b). The facing curve is defined circular and tangential to the window at the reed separation point, in accordance with common practice [4]. Geometrically, once two of the three parameters are set, the third is already fixed. Therefore, facing curve gets excluded as it becomes redundant after setting the other two parameters. Attaining to these considerations, the design aspects and related parameters used in the experiment can be listed as:

- Chamber size: The chamber is the inner cavity between the baffle and the throat (Fig. 1). The cross-section of the chamber is seen in Figure 2a. The chamber size is evaluated via the variable $s_{\text {chamber }}$, which is the shortest distance from the central axis to the chamber wall. Different chamber sizes are shown on the cross-sections in Figure 3.

- Baffle height: The inner part of the mouthpiece next to the tip is referred to as the baffle. Several baffle designs exist. The design adopted here is called a step baffle. The step baffle design has a triangular profile, resembling a stairway step. It starts from the tip rail and then drops into the chamber as shown in Figure 2c. The baffle height is defined with the variable $h_{\text {baffle }}$, as the distance of the baffle peak from the upper surface of the mouthpiece cavity, measured parallel to the tip-opening. Varying baffle heights are shown with different shades in Figure 2c. Other variables defining the baffle profile, $b_{1}$ and $b_{2}$, are kept constant.

- Throat size: The throat is the transition cavity between the chamber and the bore (Fig. 1). The
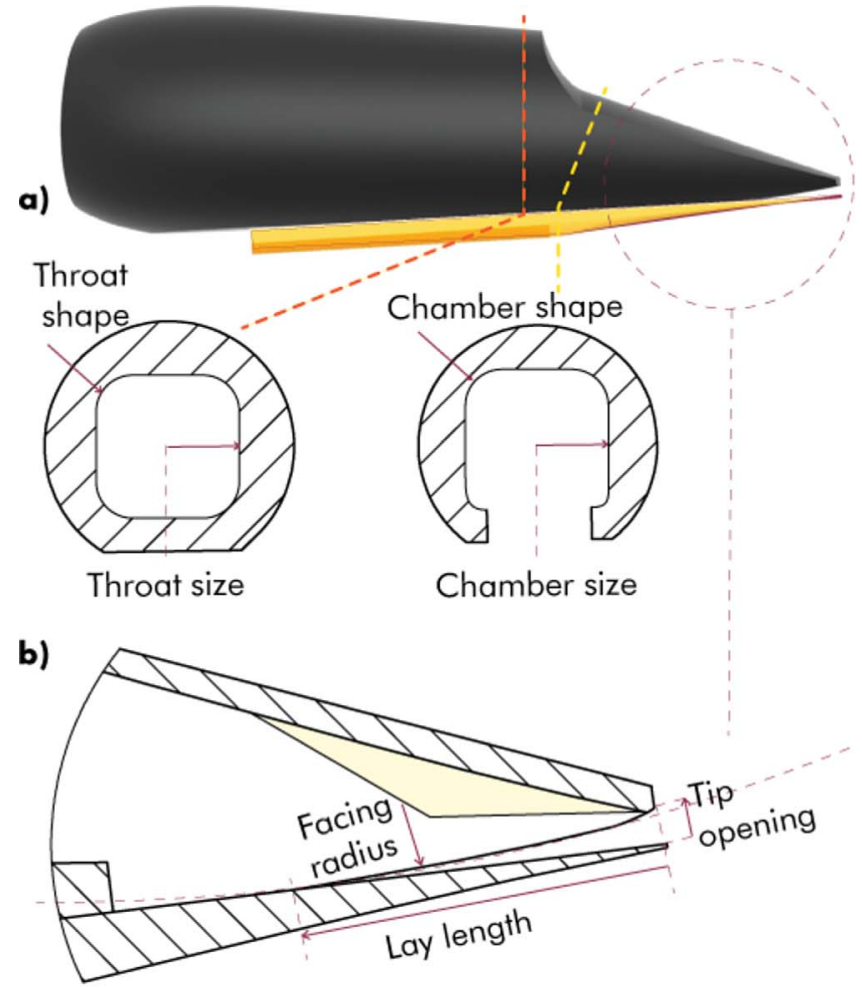

c)

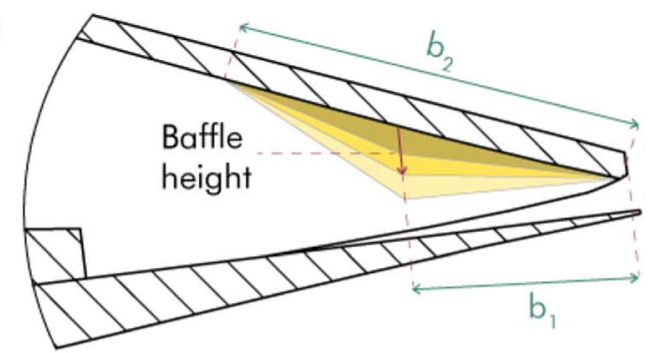

d)

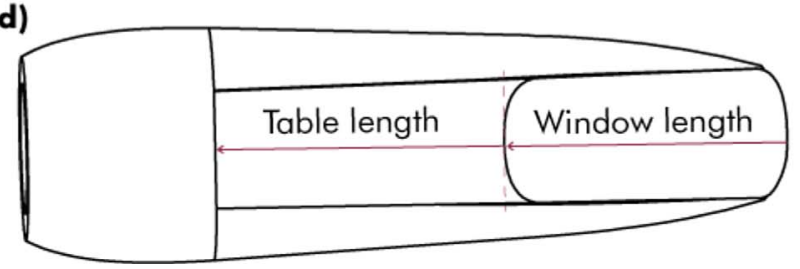

Figure 2. Design parameter definitions used in the study. The red circle in (a) highlights the location of the cross-sections in (b) and (c).

throat cross-section is seen in Figure 2a. The throat size is evaluated in the same way as the chamber size with the shortest distance from the central axis to the throat wall, $s_{\text {throat }}$. Varying throat sizes used in the experiment are shown in Figure 3.

- Throat shape: It is the shape of the cross-section, and it is defined with the variable $r_{\text {throat, }}$ which denotes the fillet radius of the cross-section area corners. Varying throat shapes are shown in Figure 3.

- Chamber shape: It is also defined as the crosssection shape, same way as the throat shape. The variable defining the chamber shape is $r_{\text {chamber }}$, which 


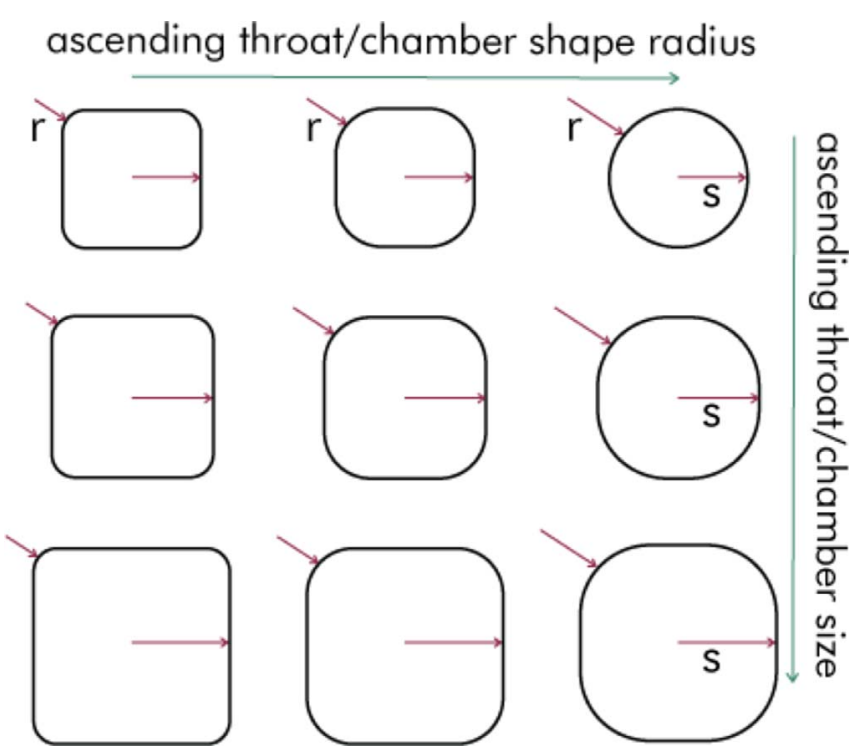

Figure 3. Throat and chamber cross-section shapes and sizes used in the study, ordered by ascending shape radius from left to right $\left(r_{\text {throat }}\right.$ and $\left.r_{\text {chamber }}\right)$, and ascending size ( $s_{\text {throat }}$ and $\left.s_{\text {chamber }}\right)$ from top to bottom.

denotes the fillet radius of the cross-section area corners (Fig. 3).

- Window length: The window is the opening between the reed and the mouthpiece. Its length $l_{\text {window }}$ is defined as the length of the centerline from the tip to the table (Fig. 2d).

- Table length: The table is the interface where the reed is fixed to the mouthpiece. Its length $l_{\text {table }}$ is measured from the end of the window to the end of the reed (Fig. 2d).

- Lay length: The lay length $l_{\text {lay }}$ is measured as the distance between the tip of the mouthpiece and the point where the reed separates from the mouthpiece, in rest position and without lip force (Fig. 2b).

- Tip opening: The tip opening $h_{\text {tip }}$ is the vertical distance between the tip of the mouthpiece and the tip of the reed, in rest position and without lip force (Fig. 2b).

\subsection{Parametric mouthpiece model}

As the literature study indicates that most of the design parameters affect multiple mouthpiece features $[4,8,12,15$, 22 , the decision on one design parameter will affect the decisions on other parameters. The most convenient way to analyze the effects of the design parameters is to change them one by one and observe the results. This requires creating a base mouthpiece design template and testing each parameter at defined levels.

To develop a design template with intervals of parameters, an existing mouthpiece design is used as a starting point. For this purpose, a Yanagisawa ebonite alto saxophone mouthpiece (\#6 with $1.83 \mathrm{~mm}$ tip opening and

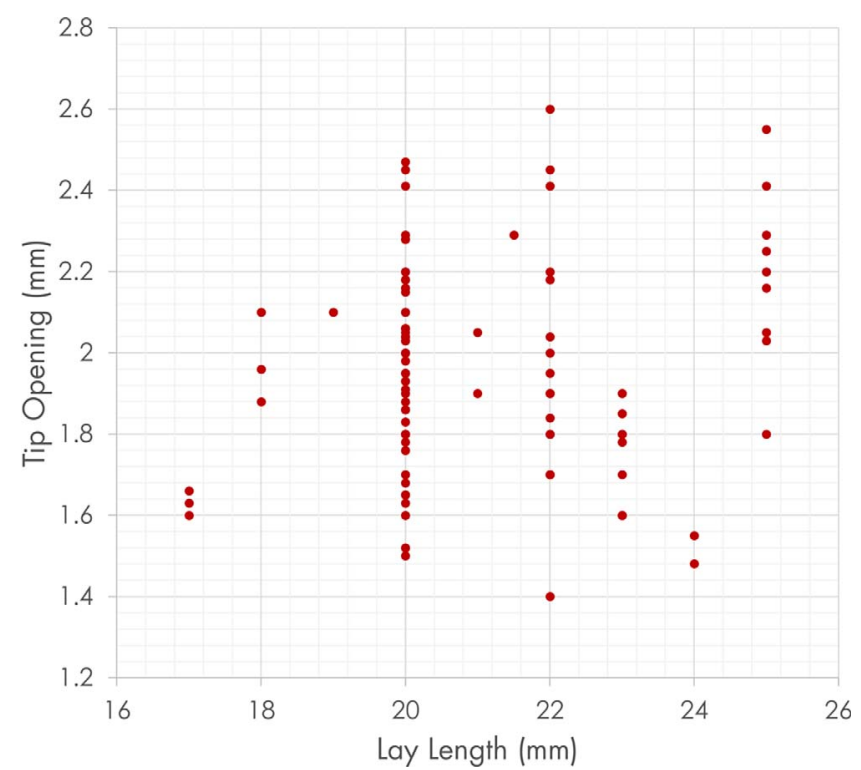

Figure 4. Tip opening and lay length values of 100 alto saxophone mouthpieces surveyed.

$22 \mathrm{~mm}$ lay length [36]) has been chosen due to being a well-established design and having design parameters at average values. The process starts with $3 \mathrm{D}$ scanning of the mouthpiece to obtain its dimensions precisely. A Konica Minolta VIVID 9i laser scanner is used for the 3D scanning, which has $0.03 \mathrm{~mm}$ Z-Depth and $0.145 \mathrm{~mm}$ X\&Y resolution. Since the scan data is a point cloud, it is processed by PolyWorks software to be converted into a mesh model. Following this, the mesh model is converted into a parametric model by the Scanto3D module of SolidWorks. To define an interval for each parameter and dynamically control these, the Grasshopper module of Rhinoceros is used, and the design template development is completed.

The ranges of the parameters used in the design template are set based on the mouthpiece designs available in the market, in order to ensure the playability of the tested mouthpieces. A survey of mouthpieces available in the market was carried out to have a reference for the variability of the parameters. This was done by considering the 100 most sold $^{1}$ alto-saxophone mouthpieces in a widely used online platform. For instance, the tip openings of these mouthpieces vary between 1.4 and $2.6 \mathrm{~mm}$, and the lay lengths are between 17 and $25 \mathrm{~mm}$, as shown in Figure 4 .

\subsection{Experiment design}

To observe the effects of design parameter changes on the selected mouthpiece features, each parameter is to be tested at different levels, and the results are to be analyzed for the responses of the parameters. If the possible interactions are also included, all possible combinations of parameters should be tested at varying levels. However, a full factorial design would yield a very high number of cases to be tested, such as for nine parameters at three levels,

$\overline{1}$ As of February 2019; http://www.thomann.de. 
the number of cases would be $3^{9}$. Neither experimenting nor analyzing such a high number of cases is practical. Therefore, a fractional factorial method to analyze the main effects and the interactions of the parameters is needed. An experimental design following the Taguchi method simplifies the statistical design by employing orthogonal arrays, which reduces the variance for the experiment with optimum settings of control parameters [14]. The use of orthogonal arrays minimizes the number of runs in the experiments. For any given pair of columns in the orthogonal array, all combinations of parameter levels occur an equal number of times. The Taguchi method provides standard orthogonal arrays based on a number of factors and levels. In this study, there are nine parameters, and to observe any nonlinearity in the output, they are tested at three levels. The L27 $\left(3^{13}\right)$ orthogonal array of Taguchi can be used to analyze up to 13 parameters with three levels using 27 runs. Therefore, it is the most suitable orthogonal array for nine parameters at three levels, with a reasonable number of experiment runs. The parameter configurations for the 27 runs are presented on Table A.1 in the Appendix.

The levels of the parameters of the experiment are chosen within the ranges identified in the parametric mouthpiece model. Critical values such as maximum or minimum are avoided, thus each level is set in a way to prevent possible interactions between the parameters or unplayable combinations, which may show misleading results. Table 1 lists the design parameters and their level descriptions. The three levels of the parameters are combined to design the 27 mouthpieces as indicated in Table A.1 in the Appendix. These 27 designs were then 3D printed using stereolithography (SLA) technology, by a Formlabs Form 2 printer, as it provides the consistency and precision needed (with dimensions within a $\pm 0.1 \mathrm{~mm}$ tolerance).

\subsection{Acoustical experimental setup and procedure}

For the acoustical assessment of the characteristics of the 27 mouthpieces, an artificial blowing machine was used [37]. Such artificial setups are commonly used in music acoustics to evaluate the physics of wind instruments independently of the player's actions [6, 38]. The artificial blowing machine allows to adjust the blowing pressure and lip position and provides repeatable conditions for the comparison of the mouthpieces. The used artificial blowing machine is based on the control of the air pressure in a $170 \mathrm{~cm}^{3}$ plexiglass box representing the oral cavity of a player, as shown in Figure 5a. This is achieved by the control of a proportional valve (SMC; type PVQ33-5G-23) at the air entrance to the cavity. The mouthpiece is inserted with the reed facing upwards and the reed meets an artificial lip consisting of a metallic rod with a rubber cover, mimicking the lower lip of the player (see [39] for more details). The artificial lip is mounted on a translation stage, that allows to regulate and fix its vertical position. The valve adjustments and the data recordings are performed via an external PC with National Instruments hardware and software.

The mouthpieces were tested individually. For each test, the mouthpiece was attached to a saxophone neck (STAGG
Table 1. Parameter level descriptions. All units are in $\mathrm{mm}$.

\begin{tabular}{lccc}
\hline & Level 1 & Level 2 & Level 3 \\
\hline$s_{\text {chamber }}$ & 6 & 7 & 8.5 \\
$h_{\text {baffle }}$ & 0 & 3 & 5 \\
$s_{\text {throat }}$ & 6 & 7 & 8 \\
$r_{\text {throat }}$ & 2 & 4 & 6 \\
$r_{\text {chamber }}$ & 2 & 4 & 6 \\
$l_{\text {window }}$ & 35 & 38 & 41 \\
$l_{\text {table }}$ & 23 & 28 & 31 \\
$l_{\text {lay }}$ & 18 & 21 & 24 \\
$h_{\text {tip }}$ & 1.6 & 2 & 2.4 \\
\hline
\end{tabular}

Alt Saxophone 77-SA) and inserted into the artificial mouth, as shown in Figure 5a, so that the artificial lip rested on the reed at $10 \mathrm{~mm}$ from the mouthpiece tip. Only the neck of the saxophone was used, as this simplified changing mouthpieces while maintaining the setup configuration, as well as improved the stability of the setup and the positioning of the external microphone. This implies that a tone at around $410 \mathrm{~Hz}$ is obtained, which is within the playing range of the alto saxophone $(138-830 \mathrm{~Hz})$. The same synthetic reed (Légère, strength 3), which is independent of humidity, was used for all mouthpieces. The acoustic pressure in the mouthpiece was recorded via a piezo-resistive pressure transducer (Endevco 8507C-2) inserted to the mouthpiece via a lateral orifice, at $5.7 \mathrm{~cm}$ from the tip, included in the 3D-print design. The acoustic pressure in the artificial mouth was measured with a pressure transducer (Technoterm 5400, with a resolution of $1 \mathrm{~Pa}$ ). This artificialmouth pressure value was used in a feedback loop as a control parameter to establish the pressure patterns to be used during the tests and regulate the settings of the air valve. A second piezo-resistive pressure transducer (Endevco $8507 \mathrm{C}-2$ ) was placed at $5 \mathrm{~cm}$ in front of the saxophone's neck output to measure the external sound.

For the measurements concerning resistance, loudness, and brightness, an ascending pressure $(1 \mathrm{kPa} / \mathrm{s})$ was used to drive the blowing pressure in the artificial mouth, as shown at the top of Figure 6. Notice that the pressure in the artificial mouth increases at $1 \mathrm{kPa} / \mathrm{s}$ before the tone onset, but faster during the transient. This jump is due to a change in the acoustic impedance at the tip of the reed when the reed starts to oscillate. The test was repeated three times, to ensure the soundness of the results and avoid any inconsistencies due to the experiment setup. This data is provided in the Appendix, Figure A.1. The mean value considering the three repetitions at each of the 27 mouthpieces is used in the analysis.

For the measurements concerning flexibility, a complete alto saxophone was used (STAGG Alto Saxophone 77-SA). The mouthpieces were equipped with two pressure transducers (Endevco 8507C-2), as shown in Figure 5b, to measure the mouthpiece pressure and the blowing pressure in a real playing configuration [33]. The same synthetic reed and neck as in the artificial configuration were used. For this task, the first author tested all mouthpieces in a blind test, where the mouthpieces were randomly assigned with a new 
numeration. The player performed pitch adjustments while fingering the tone $\mathrm{C} \#_{5}$ (concert pitch $\mathrm{E}_{5}, 659.26 \mathrm{~Hz}$ ) with all the mouthpieces. The test was repeated three times. The reported value is the widest pitch variation interval played.

\section{Data processing and analysis \\ 4.1 Acoustical characteristics extraction}

In order to analyze the relationship between the 9 selected geometry parameters and the acoustical characteristics of every tested mouthpiece, four mouthpiece features have been tested. To do that, the recorded signals were processed and the following features were analyzed:

- The difficulty of blowing into a mouthpiece to create a sound, i.e., the "resistance" it provides when beginning a tone, is assessed by measuring the threshold of oscillation of every mouthpiece. The threshold of oscillation is obtained as the minimum air pressure in the artificial-mouth $\left(p_{\text {mouth }}\right)$ required to start a tone [6]. This is found as the artificial-mouth pressure value corresponding to a mouthpiece pressure amplitude of $p_{\text {mouthpiece }}=0.5 \mathrm{kPa}$ at the tone onset (R in Fig. 6), a value that is above the noise level for all mouthpieces and right at the beginning of the oscillations.

- The "loudness" of every mouthpiece is assessed as the external sound level that is achieved at a certain artificial-mouth pressure. Such measurement relates to the acoustic efficiency of the instrument, since it considers the relationship between the input pressure and the output pressure across the system. For that, an artificial-mouth pressure of $p_{\text {mouth }} \in[12,12.5] \mathrm{kPa}$ was selected, since at this level all mouthpieces would produce a sound in the stationary state. Within this inter$\mathrm{val}$, the average amplitude of oscillation of the external sound signal was calculated. This was obtained as the difference between the mean upper envelope and the mean lower envelope during the interval (L in Fig. 6).

- The "brightness" of a sound is a perceptual feature that is related to the distribution of energy across the partials of its spectrum, and it is often assessed as the spectral centroid of the sound $[18,40]$. The spectral centroid represents the center of gravity of the signal spectrum [41]. To extract the spectral centroid corresponding to the sound of the tested mouthpieces, a segment of $300 \mathrm{~ms}$ of the mouthpiece pressure signal recorded at the maximum achieved blowing pressure was selected for every mouthpiece (B in Fig. 6). In case the reed closed against the mouthpiece during the test (at a blowing pressure below $15 \mathrm{kPa}$ ), the selected segment is located before the release transient, i.e., during the steady part of the recorded sound and right before the sound ended. For the mouthpieces that did not close (because their extinction threshold was higher than $15 \mathrm{kPa}$, and posing a safety risk) the selected segment is located at a blowing pressure of $15 \mathrm{kPa}$ (see $\mathrm{B}$ in Fig. 6).

\section{a) Mouthpiece Lip Pressure transducer}

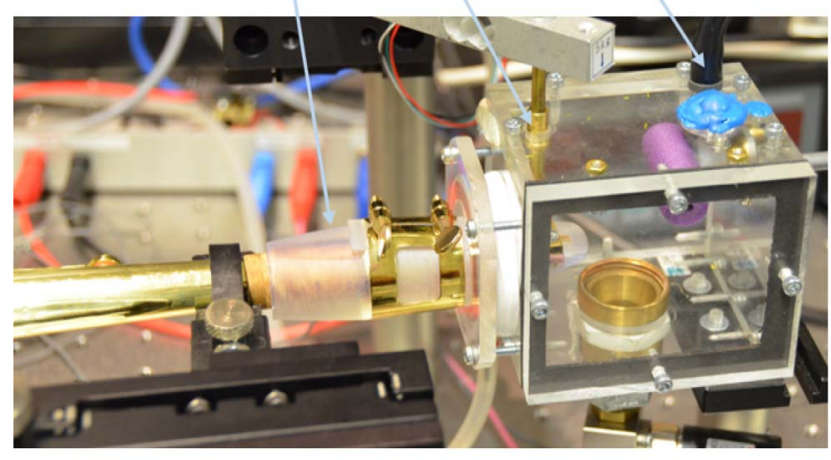

b)

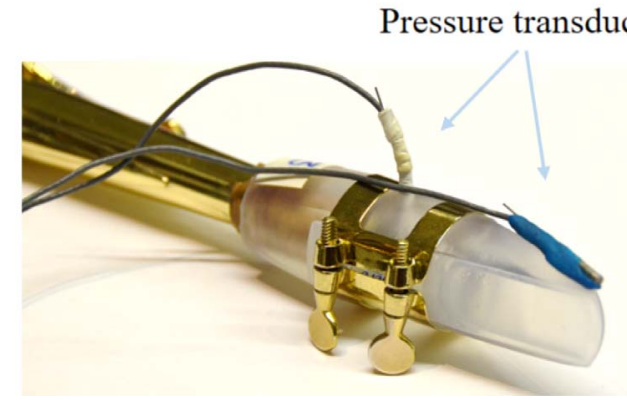

Figure 5. (a) Artificial mouth used to blow into a 3D-printed saxophone mouthpiece. (b) Pressure transducers attached to a saxophone mouthpiece to measure pressure variations in the mouthpiece and in the mouth of a player.

Hence, this calculation considers that differences in the beating conditions might appear, which result in differences in brightness [42]. After smoothing the segment with a Hanning window and filtering it at $15000 \mathrm{~Hz}$ to reduce high-frequency noise, the first 35 peaks of its spectrum were used to compute a peak-wise spectral-centroid as in [41]. Figure 7 shows the spectra of the sound produced by two mouthpieces that present low and high spectral centroids.

- To assess "flexibility", the interval of pitch variation $\left(I_{\text {pitch }}\right)$ was obtained as the frequency difference from the highest to the lowest pitch during the performance of pitch adjustments while fingering the tone $\mathrm{C} \#_{5}$ (concert pitch $\mathrm{E}_{5}, 659.26 \mathrm{~Hz}$ ), as shown in Figure 8.

Notice that the available recorded data during the performance of the ascending ramp in $p_{\text {mouth }}$ defined the manner in which resistance, loudness and brightness were assessed, in order to obtain a standard procedure across all mouthpieces. A compromise was made to compare the mouthpieces at similar playing conditions: at the tone onset for resistance, at a certain blowing pressure in the steady state for loudness, and at the maximum achieved blowing pressure for brightness. The four selected acoustical properties were then analyzed independently of each other as follows.

\subsection{Statistical analysis}

The analysis of the Taguchi method is largely focused on orthogonal arrays and analysis of variance (ANOVA). The aim of the experiment was to determine which parameters 

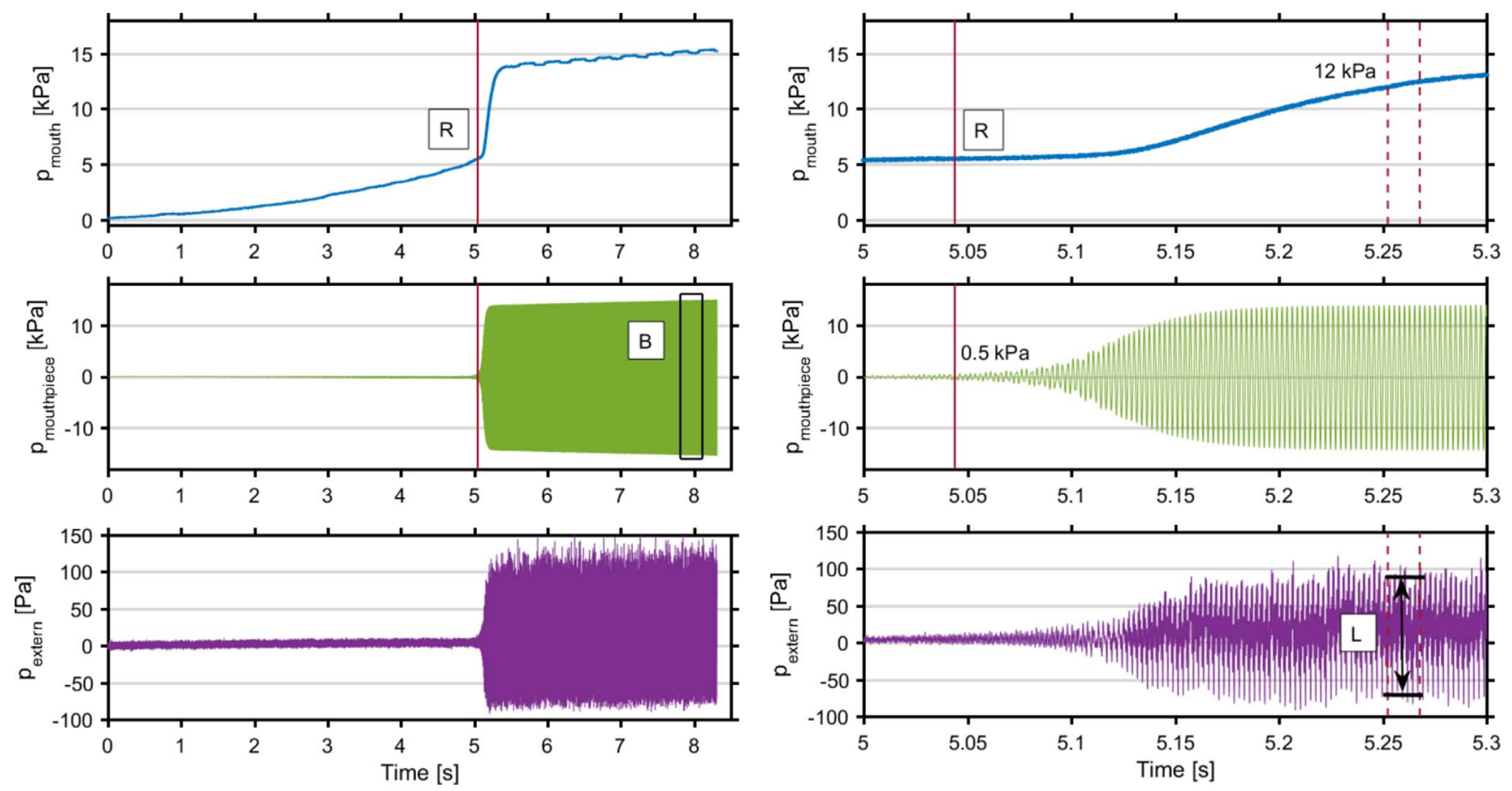

Figure 6. Artificial-mouth pressure $p_{\text {mouth }}$, mouthpiece pressure $p_{\text {mouthpiece }}$ and external sound pressure $p_{\text {extern }}$ when recording a mouth-pressure ascending ramp at $1 \mathrm{kPa} / \mathrm{s}$. On the right side, zoomed view of $300 \mathrm{~ms}$ around the tone onset. $R$ indicates the threshold of oscillation, considered to assess resistance (vertical red line indicates the first instant where $p_{\text {mouthpiece }}>0.5 \mathrm{kPa}$ ). $B$ indicates the time interval of mouthpiece pressure used to compute the spectral centroid (Fig. 7), considered to assess brightness. $L$ indicates the external sound amplitude considered to assess loudness, at $p_{\text {mouth }}$ between 12 and $12.5 \mathrm{kPa}$. [Mouthpiece no. 10, first repetition].

have influence on which features and at what amount. The influence of the parameters is observed on the main effect plots of data means (Fig. 9). The plots demonstrate the mean response at each level of the parameters. As each level of a parameter appears 9 times, the mean response of those 9 measurements is analyzed for the main effects. The validity of the main effects is verified with the statistical significance of every effect in the ANOVA analysis. The ANOVA results for the four experiment cases are presented in Tables A.2, A.4, A.6 and A.8 in the Appendix. A stepwise model reduction is applied according to statistical significance criteria [43]. For the model reduction in the cases of oscillation threshold, spectral centroid and pitch variation interval, a significance level of 0.05 , i.e., a confidence level of $95 \%$, is taken for the $p$-value. In the case of external sound level, the $p$-value threshold is taken as 0.15 . At each step of model reduction, the parameter with the highest $p$-value is eliminated, and the ANOVA is repeated. The reduction is followed until all the parameters are within the taken $p$ value threshold. The ANOVA results after reduction are presented in Tables A.3, A.5, A.7 and A.9 in the Appendix.

Based on the ANOVA results, the effective parameters for each mouthpiece feature, to be used in mouthpiece personalization, are determined. The statistically significant parameters for the four experiment cases are highlighted in Figure 9. In the following section, the results of the analysis are discussed, and the effective parameters for each feature to be used in the mouthpiece personalization model are presented.

\section{Influence of design parameters on mouthpiece features}

The influence of each of the 9 selected design parameters on the 4 tested mouthpiece features (i.e., on the extracted acoustical characteristics) is observed via main effect plots (Fig. 9). The obtained values of the 4 features for every tested mouthpiece are shown in the Appendix (Fig. A.1), with indication of the measurements obtained in the repeated tests.

Changes in $l_{\text {window }}$ and $l_{\text {table }}$ did not demonstrate any meaningful effect on any of the measurements. Besides, both had very low statistical significance values in ANOVA for all four features measured. Therefore, it is safe to conclude that these two parameters may be excluded in the resultant mouthpiece personalization model. Yet it is to be noted that this conclusion is valid within the scope of the experiment as well as within the tested values. These two parameters might still have an influence on other mouthpiece characteristics that are not covered in this work.

\section{Resistance}

The initial ANOVA results of oscillation threshold measurements are presented in Table 4. Main effect plots for parameter means are in Figure 9a. As seen in the plot, $s_{\text {throat }}, r_{\text {throat }}, l_{\text {window }}$ and $l_{\text {table }}$ do not present any significant effect on the oscillation threshold. These parameters also 
have very low statistical significance, as seen in the initial ANOVA results (Table 4).

The model is reduced by eliminating the parameter with the highest p-value at each step until all the parameters are within the 0.05 threshold (Table 5). As expected, $s_{\text {throat }}$, $r_{\text {throat }}, l_{\text {window }}$ and $l_{\text {table }}$ are not in the reduced model. The most influential parameters on resistance are $h_{\text {tip }}$ and $l_{\text {lay }}$, both having a confidence level of $100 \%$. This is very much in line with the literature review $[4,7,12]$, since the oscillation threshold is proportional to the tip opening and to the reed stiffness [44]. The influence of the lay length relates to the modification of the vibrating length of the reed, as well as the way the reed curls upon the lay, and hence affects the reed stiffness [8-10]. The parameters tip opening $h_{\text {tip }}$ and lay length $l_{\text {lay }}$ define the airflow cross-sectional area between the player and the mouthpiece, hence directly related to airflow resistance. $s_{\text {chamber }}, r_{\text {chamber }}$ and $h_{\text {baffle }}$ showed similar effects to each other on the results, also appearing to be effective between two levels only (Fig. 9). These three parameters define the size of the inner cavity of the mouthpiece, which justifies their effect on the oscillation threshold [12]: the internal geometry of the mouthpiece affects the airflow characteristics and the output impedance of the mouthpiece [45], and hence it has an important role on the sound production [22]. As a result, one can conclude that the oscillation threshold is affected by $h_{\mathrm{tip}}, l_{\text {lay }}, s_{\text {chamber}}$, $r_{\text {chamber }}$ and $h_{\text {baffle. }}$

\section{Loudness}

ANOVA results on the mean response of external sound level are presented in Table 6 in the Appendix A. After model reduction (Table 7 ), only $s_{\text {throat }}$ and $h_{\text {tip }}$ remained as statistically significant.

The throat size $s_{\text {throat }}$ appeared to be the dominant parameter for the loudness of the instrument, with a confidence level of $96 \%$ ( $p=0.004$, Table 7$)$. $s_{\text {throat }}$ shows an inversely proportional relationship to external sound level (Fig. 9b). $s_{\text {chamber }}$ also shows a similar inversely proportional effect, from which one can reach a generic conclusion that reducing the cross-sectional area of the internal cavity may increase the loudness of the mouthpiece. Whereas, the tip opening $h_{\text {tip }}$ demonstrated inverse trends on either side of level 2 (Fig. 9b). This indicates that the highest loudness is achievable with an average $h_{\text {tip }}$ value. A probable reason for such behavior is that, with small $h_{\text {tip }}$, when high blowing pressure is applied to the mouthpiece, the reed closes against the mouthpiece, blocking the airflow. On the other hand, with large $h_{\text {tip }}$, playing the mouthpiece requires higher blowing pressure and hence it is more difficult to achieve louder sounds. Consequently, the external sound level is defined by the parameters $s_{\text {chamber }}, s_{\text {throat }}, r_{\text {chamber }}$, $l_{\text {lay }}$ and $h_{\text {tip. }}$.

\section{Brightness}

Initial and reduced model ANOVA results for spectral centroid measurements are provided in Table 8 and Table 9 in the Appendix A, respectively. After the stepwise reduction of statistically insignificant terms, $h_{\text {baffle }}, s_{\text {throat }}$ and $l_{\text {lay }}$ were the remaining parameters. The lay length $l_{\text {lay }}$ is the most prominent parameter, with a $91 \%$ confidence level. This correlation between $l_{\text {ay }}$ and brightness has not been reported in the literature. It is however expected that changes in $l_{\text {ay }}$ may affect reed beating and hence brightness $[9,42]$. Both the baffle height $h_{\text {baffle }}$ and the throat size $s_{\text {throat }}$ were expected to have an influence on the spectral centroid [11, 46], as seen in Figure 9c, yet they have unexpected trends between levels 1 and 2. One potential source of this trend is the existence of an interaction between the parameters. In conclusion, $l_{\text {lay }}, h_{\text {baffle }}$ and $s_{\text {throat }}$ are found to be effective parameters on spectral centroid.

\section{Flexibility}

The measurement of flexibility is defined in this study as the interval between the highest and the lowest pitch achievable by the player without changing fingering $\left(I_{\text {pitch }}\right.$ in Fig. 8). The initial ANOVA results and eventual results after model reduction are presented in Table 10 and Table 11 in the Appendix A. $s_{\text {throat }}, r_{\text {throat }}, r_{\text {chamber }}, l_{\text {window }}$, $l_{\text {table, }}$ and $l_{\text {lay }}$ were eliminated at each reduction step as statistically not significant terms. They also demonstrate remarkably less effect on the main effects plot (Fig. 9d). $h_{\text {baffle }}$ and $s_{\text {chamber }}$ are the most influential parameters with $99 \%$ and $91 \%$ confidence levels, respectively.

The baffle height $h_{\text {baffle }}$ and the chamber size $s_{\text {chamber }}$ define the first cavity of the mouthpiece, significantly influencing the impedance at the beginning of the air column $[45,46]$. In this study, both parameters show a similar trend: a wider cavity (low $h_{\text {baffle }}$ and high $s_{\text {chamber }}$ ) results in a reduced possibility to adjust pitch. The tip opening $h_{\text {tip }}$ appeared to be influential only between level 1 and 2 . A probable explanation to this observation is the usage of the same reed (i.e., same reed strength) for all the experiment mouthpieces. Combining different reed strengths might imply differences in lip force, which would affect $h_{\text {tip }}$ and would yield to diverse results. Consequently, pitch interval is affected by the parameters $h_{\text {baffle }}, s_{\text {chamber }}$ and $h_{\text {tip }}$.

\section{Personalization model and user study}

The experiment results provided an indication of the relations between mouthpiece design parameters and features related to performance. The direction and the relative size of the parameter effects are deducible from the results, as seen in the plots in Figure 9. The ultimate goal was to tailor the mouthpiece to the needs of saxophone players by devising a mouthpiece personalization model. The outcome of the experiment is used to build an initial personalization model, which has been covered in previous work [13]. The personalization model operates based on the relationships obtained in the experimental analysis (Sect. 5). These relationships are adopted as a proxy to estimate the features of the mouthpiece. More specifically, the parameter configurations providing the extrema of each feature are deduced from the experiment results (for example: the 

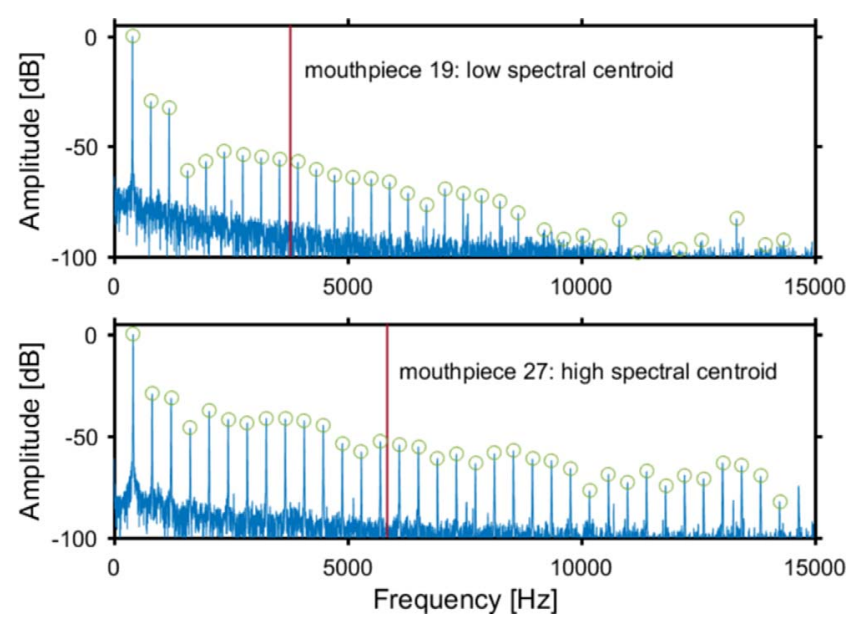

Figure 7. Spectra of the mouthpiece-pressure signal comparing two mouthpieces with low and high spectral centroid (marked as a vertical line). The spectral centroid is calculated by considering the amplitude of the first 35 peaks of the spectrum (marked with circles).

maximum brightness is expected when $h_{\text {baffle }}$ is set at level $3, s_{\text {throat }}$ at level 2 and lay at level 3 ). The feature extrema form the boundaries of the scale, in which a feature can be configured. Relative to either end of the scale, the parameter values are iterated to obtain the desired performance. Moreover, eight mouthpieces manufactured with configurations corresponding to the extrema provide a reference for the players to personalize their own mouthpieces. The validity of this personalization model is verified with a user study, which is covered further in this section. Notice that the proposed personalization model is not predicting absolute values, but relatively estimating the features of the mouthpiece. This is why a user study is a sound approach to test the model and provide a proof of concept with the end-users.

\subsection{Personalization model}

Table 2 shows the resultant dependence matrix between mouthpiece features and parameters. In addition to the dependencies identified in the analysis of the experiments (Fig. 9), the relationship between loudness and $h_{\text {baffle }}$ is also included in the personalization model, even though it was above the $p$-value threshold ( $p=0.232$ for $h_{\text {baffle }}$ in the analysis of external sound level, at the second step of the model reduction). To build a reliable model, the subject matter knowledge is customarily used to interpret the results $[43,47,48]$. In this case, the motivation of including the $h_{\text {baffle }}-$ loudness relationship is that the effect of baffle height on the loudness of the mouthpiece (also stated as power) has been reported by players [4] and manufacturers [49]. Furthermore, the impact of baffle has been reported to produce observable changes in the mouthpiece impedance [46].

As seen in Table 2, the dependence matrix shows a tightly coupled design with parameters affecting multiple features. The performance requirement of a mouthpiece is defined by the players' request regarding these features.

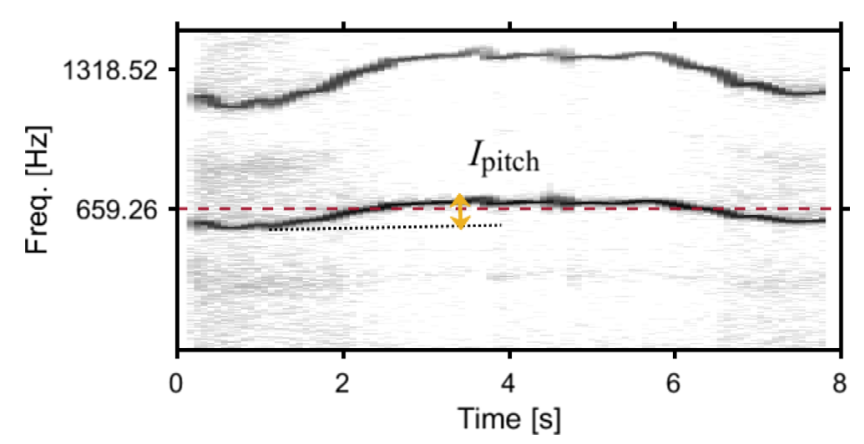

Figure 8. Spectrogram of the mouthpiece pressure during the performance of pitch adjustments when fingering the tone $\mathrm{C}_{5}$ (concert pitch $\mathrm{E}_{5}$ ) showing the theoretical fundamental frequency $(659.26 \mathrm{~Hz}$; marked as a horizontal dashed line) and the first harmonic. The interval of pitch variation $\left(I_{\text {pitch }}\right)$ is considered between the lowest and the highest fundamental frequency achieved during the task. [Mouthpiece no. 6, first repetition].

Hence, the inputs to the model are four features. This results in a mathematical problem with 4 equations and 7 unknowns. As this is unsolvable, a novel approach is needed.

To deliver a certain feature performance, there are multiple solutions with different parameter configurations. As an example, considering flexibility, a high $s_{\text {chamber }}$ and high $h_{\text {baffle }}$ combination may yield to the same result as low $s_{\text {chamber }}$ and low $h_{\text {baffle }}$ (Fig. 9). Therefore, both configurations would be valid for the given flexibility requirement. However, the features affected by common parameters are dependent. In other words, while both configurations provide the same result for flexibility, they have different effects on other features. Thus, it is essential to select an appropriate parameter configuration to provide the desired feature requirements.

The parameter configurations for the extrema of each feature can be deduced from Figure 9. For instance, the highest flexibility would be provided by $s_{\text {chamber }}$ and $h_{\text {tip }}$ at level 1 , and $h_{\text {baffle }}$ at level 3 ; while the lowest one would have $s_{\text {chamber }}$ at level $3, h_{\text {tip }}$ at level 2 , and $h_{\text {baffle }}$ at level 1 . Therefore, these are the upper and lower boundaries within which a mouthpiece can be configured for flexibility. Therefore, players may configure the features between these upper and lower boundaries using sliders (Fig. 10). The parameter values are then fine-tuned to match the request of the player. The influence of the parameters from level 1 to 2 and from 2 to 3 are assumed linear, as in Figure 9. Once a feature is set, other features affected by common parameters are then restricted to have values in a narrower interval. Hence, the design space changes with each feature input and all 4 features cannot be set simultaneously.

The proposed model is based on the hierarchical input of features. Musicians may have a different priority on the proposed mouthpiece features. By letting them choose the most important one to be set in the first step, they are given the largest design space for this prioritized feature. The design algorithm iterates the parameters at each step of the input, with the principle of using the least number of 
a) Resistance

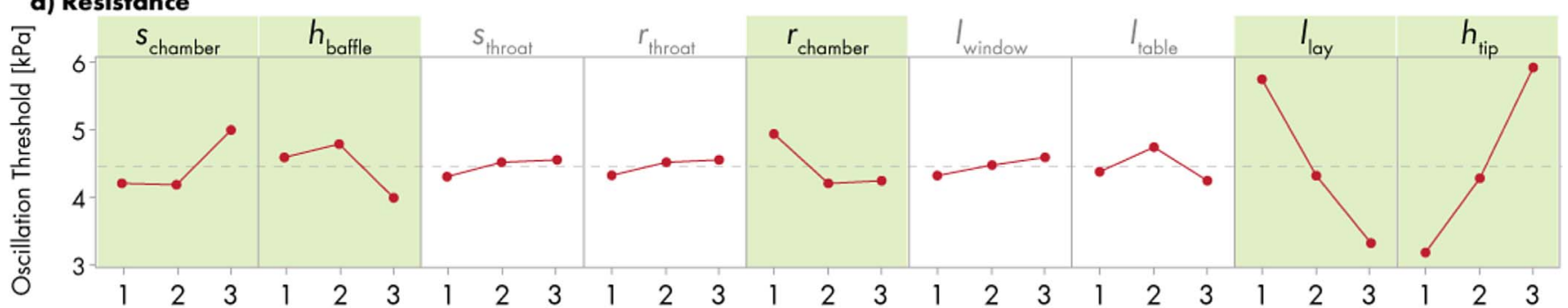

\section{b) Loudness}

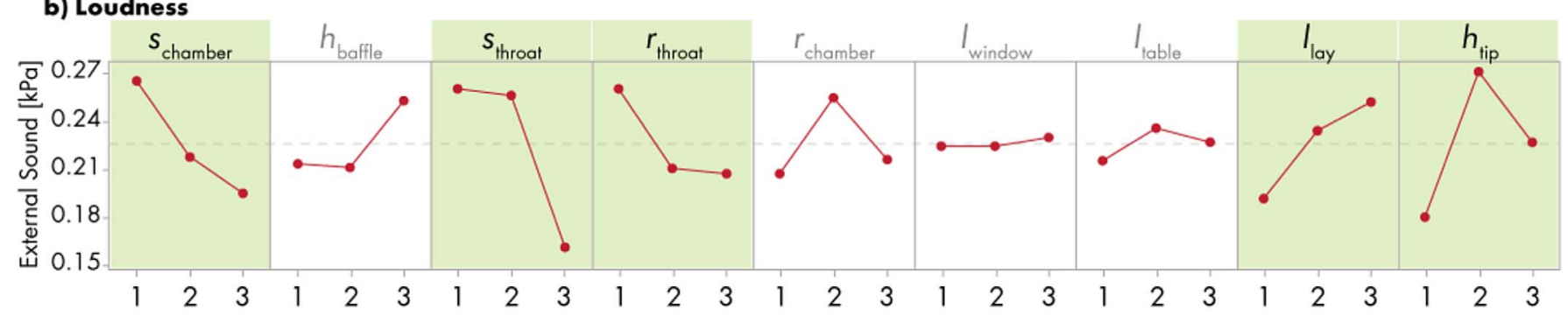

c) Brightness

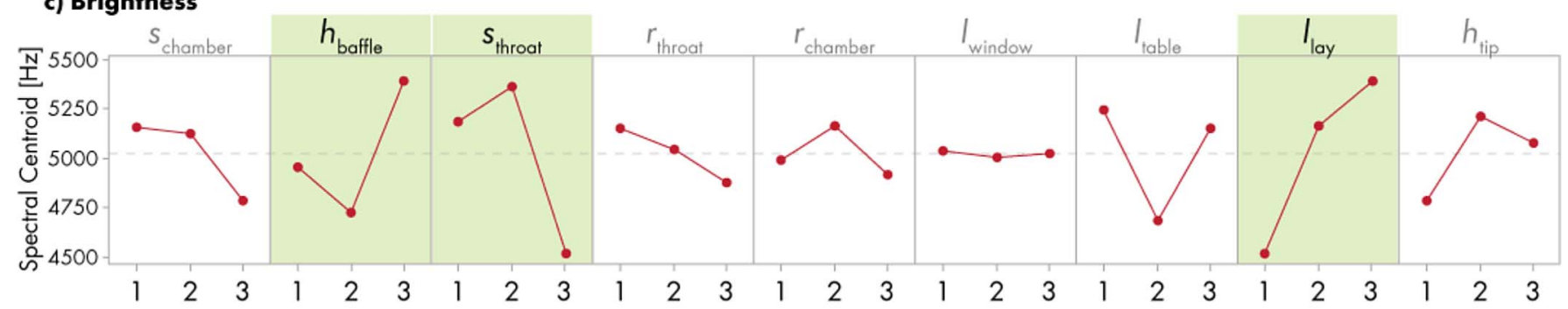

d) Flexibility

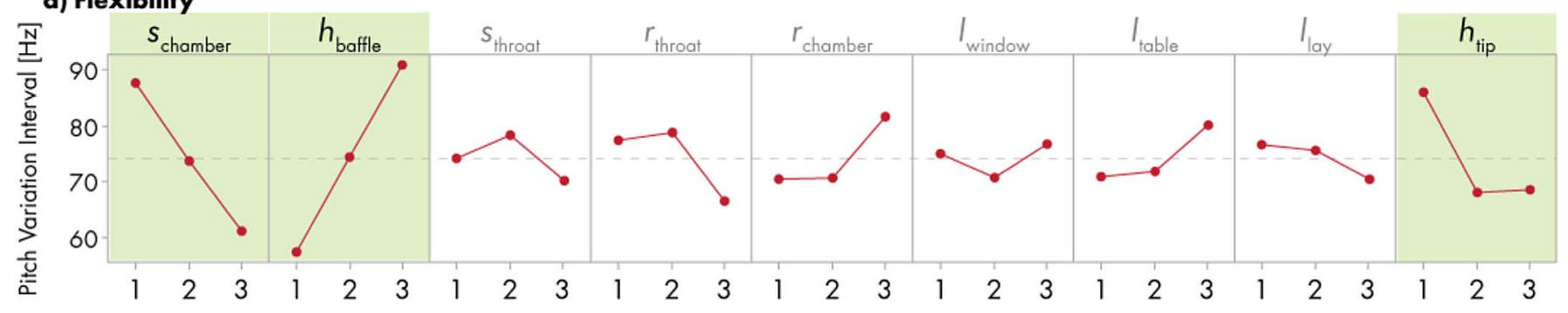

Figure 9. Main effects plots of mean of means for design parameters, showing the four experimental cases (resistance, loudness, brightness, flexibility). The horizontal axis represents the levels of parameters (1-3 levels correspond to ascending values, as in Table 1), and the vertical axis represents the mean response of 9 measurements at the given level for the measured acoustical characteristics. Statistically significant parameters after stepwise model reduction are written in bold, and highlighted in green.

parameters, thus providing the largest design space [13]. The input from saxophone players is taken via a graphical user interface (GUI), as seen in Figure 10. When the player sets a feature using sliders, the algorithm determines and shows the updated intervals for other features. Once the player reaches a desired trade-off, the final design parameters are set.

\subsection{User study}

The experiment results demonstrated how design alterations affect the performance of the mouthpiece in a quantified manner. However, it is also important to understand how saxophone players perceive these performance changes and whether their perception is in accordance with the reported experiment results. For this purpose, a user study was devised to verify the results employing the proposed personalization model.

The study was conducted with 5 participants at The Royal Conservatoire of Antwerp (Antwerp, Belgium). All participants were master level saxophone students with minimum 10 years of experience. The tests were performed with one user at a time, and each session took 40-60 min. The participants were informed about the experimental procedure and gave their consent about their anonymous participation in this study.

The user tests were carried out in two phases (Fig. 11). In the initial phase, players could test the mouthpieces, configure a personalized mouthpiece and get familiar with the mouthpiece feature changes. For this purpose, mouthpiece 
Table 2. Dependence matrix of features and parameters. The dependencies are indicated by X, and quantified by the highlighted plots in Figure 9.

\begin{tabular}{|c|c|c|c|c|c|c|c|}
\hline Resistance & $\mathrm{X}$ & $\mathrm{X}$ & & & $\mathrm{X}$ & $\mathrm{X}$ & $\mathrm{X}$ \\
\hline Loudness & $\mathrm{X}$ & $\mathrm{X}$ & $\mathrm{X}$ & $\mathrm{X}$ & & $\mathrm{X}$ & $\mathrm{X}$ \\
\hline Brightness & & $\mathrm{X}$ & $\mathrm{X}$ & & & $\mathrm{X}$ & \\
\hline \multirow{2}{*}{ Flexibility } & $\mathrm{X}$ & $\mathrm{X}$ & & & & & $\mathrm{X}$ \\
\hline & $s_{\text {chamber }}$ & $h_{\text {baffle }}$ & $s_{\text {throat }}$ & $r_{\text {throat }}$ & $r_{\text {chamber }}$ & $l_{\text {lay }}$ & $h_{\mathrm{tip}}$ \\
\hline
\end{tabular}

design configurations at levels providing the highest and lowest of each feature were manufactured. As explained in the previous section, these configurations are deduced from Figure 9 using the maxima and minima of the plots. These benchmark mouthpieces were used as a reference for the players to understand the boundaries given for each feature. The GUI in Figure 10 was also presented to the players. They could use sliders to specify how they would prefer the features in a personalized mouthpiece for themselves. The parameters corresponding to the benchmark mouthpieces were presented at the extremities of the sliders. For each feature, players tested the benchmark mouthpieces several times and specified a preference on the GUI. Each player was provided a new Vandoren V16 reed (strength 2.5) during the tests. In two cases where players could not perform comfortably with the provided reed strength, they were allowed to switch to a softer reed (strength 2). Since both of these cases were while performing with the highest resistance benchmark mouthpiece, players could still assess and compare the given mouthpieces in terms of resistance. It is further assumed that small changes in the reed properties would contribute in a similar fashion to changes across players (embouchure and vocal tract).

The second phase of the tests was performed after a personalized mouthpiece had been manufactured for each player. In this phase, players were presented with the previous benchmark mouthpieces and a new personalized one, and performed a blind comparison. To avoid long exhausting sessions that could have influenced their judgments, they were asked for their choice of the two most important features and comparisons were done only for these two features. For each feature, they were given three mouthpieces to test, and asked to rank these mouthpieces according to their judgment of how the mouthpieces perform a certain feature (e.g., from lowest to highest resistance). They were also asked to pick a favorite mouthpiece for each of the two features.

In the blind comparisons of mouthpieces for each given feature, 7 out of 10 times (i.e., 2 features and 5 players) users ranked the mouthpieces in accordance with the experiment results. Out of these 10 rankings, in 8 instances, the mouthpiece that participants picked as their favorite for the given feature was the participants' personalized mouthpiece. Brightness appeared to be the most important feature for all participants, as it was always ranked first.

After these two comparisons, they were asked to pick a favorite mouthpiece, again blindly, among all the mouthpieces tested. This was a selection among 5 mouthpieces, 4 for benchmarking and a personalized one. At the end of the second phase, 4 out of 5 participants picked their personalized mouthpieces as their favorite in the blind test.

After the blind comparison part, the personalized mouthpiece was revealed to the participants. They were asked to play the personalized mouthpiece again and rate their satisfaction about each feature on a 5-point Likert scale (from "very unsatisfied" to "very satisfied"). Figure 12 presents the levels of satisfaction of the players with their personalized mouthpieces for each mouthpiece feature.

Participants also provided feedback on the personalization model. There was consensus about personalizing a new mouthpiece based on their current mouthpiece, instead of using benchmark mouthpieces. Moreover, they reported that they would also prefer to either choose the tip opening and lay length themselves, or set them at similar values to their current mouthpiece.

\section{Discussion}

The findings disclose that each mouthpiece feature is affected by certain design parameters at relatively different quantities. It is important to observe how changes in different parameters affect the performance of the mouthpiece in different ways. Besides, another challenging task is to quantify these changes objectively using an artificial blowing machine, reducing subjectivity due to the effect of the player's vocal tract or playing habits. Therefore, the results of this study achieved more explicit guidance in mouthpiece design (e.g., description of feature-parameter relationships), in comparison to the existing literature. Furthermore, the real playing tests to measure flexibility cared for objectivity both by performing the test blindly and by considering the quantitative pitch-adjustment measurement without the subjective assessment of the player. It should be noted, however, that the blowing system reproduces the real conditions with limitations. These limitations concern the fixed mouth cavity -thus disregarding vocal tract modifications [50]- and the fixed position of the artificial lip. On the other hand, the use of such a system allows repeatable excitation. It was observed that measurement errors -which could be introduced by slight changes in the lip force against the reed- were significantly smaller than the effects due to changes in mouthpiece design (Fig. A.1), thus achieving the required reproducibility. It should also be noted that the given results are valid for the starting mouthpiece design and the way parameter intervals are designed, as well as for the chosen reed and experimental conditions. Therefore, the numerical results 


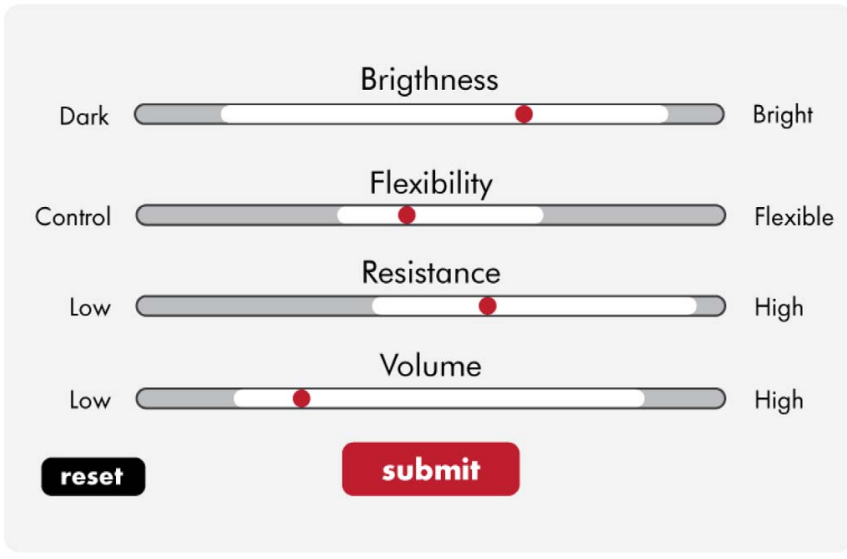

Figure 10. GUI used in the personalization process to collect the user preferences of mouthpiece features. White areas in the slider indicate the available range of the feature at that instant.
Phase I

3D printing

benchmark mouthpieces

briefing participants

participants playing

benchmark mouthpieces

for each feature

selection of preferred amount on the GUI

further trade-offs between features (if necessary)

\section{blind test}

\section{$\times 4$}

$\times 2$

\section{Phase II}

3D printing personalized mouthpieces

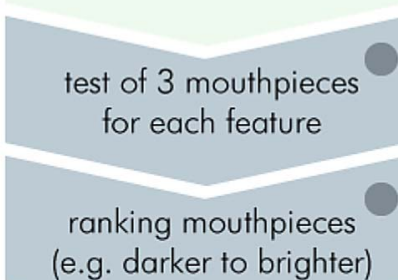

(e.g. darker to brighter)

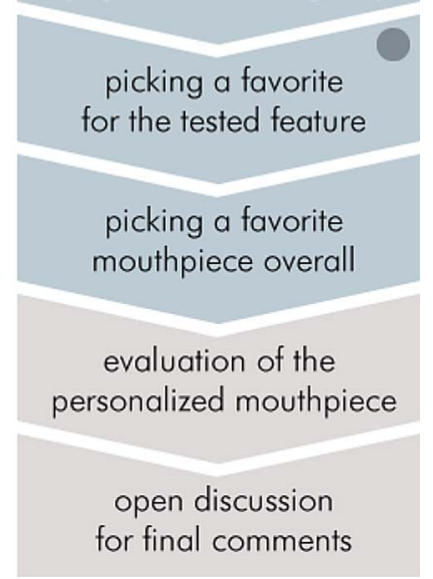

Figure 11. User study procedure.

are valid within this scenario. Nevertheless, the experiment results and reported feature-parameter relationships still provide solid evidence about the consequences of design changes on the mouthpiece.

Most of the observed feature-parameter interactions are in line with the literature, only with a few exceptions. For instance, to the best of our knowledge, the relation between $l_{\text {lay }}$ and brightness had not been reported before, and yet the experiment results show a strong correlation between them. On the other hand, $l_{\text {lay }}$ has been associated with flexibility

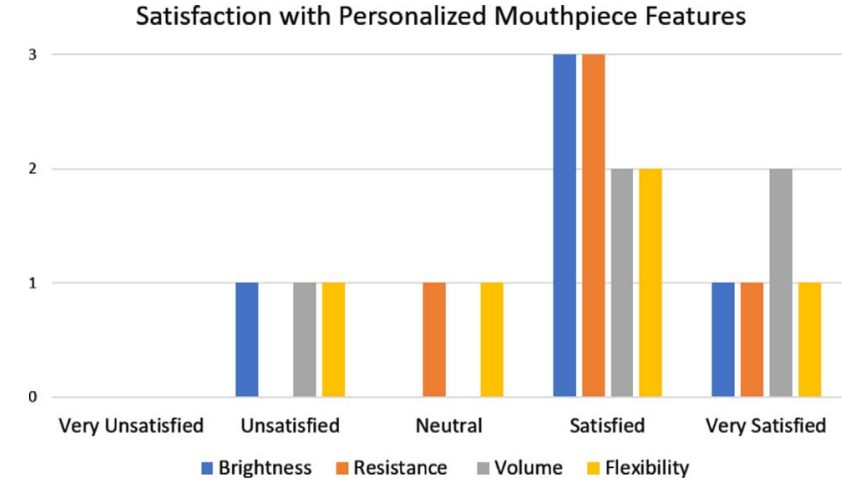

Figure 12. Survey results of players' feedback on the personalized mouthpiece features.

[20], but the results show no significance between $l_{\text {lay }}$ and flexibility. One explanation is that $l_{\text {lay }}$ was an overlooked parameter in the previous studies, which mostly focus on the parameters of the internal cavity of the mouthpiece. Another plausible explanation for the flexibility case is that the reed strength was fixed in the experiment, and lay may have an interaction with reed strength. Brightness is commonly considered being defined by chamber, throat, and baffle. The results confirm the effect of modifications in the throat and baffle design, but not in the chamber, which is both smaller and not statistically significant. Here an interaction between the chamber and throat is likely since they are successive internal cavities. In future investigations, changing only one of these parameters or combining them into a new parameter may provide more conclusive results.

Some parameters appear to be effective within certain ranges. For instance, changing the chamber size between levels 1 and 2 does not affect the resistance, but it affects loudness and flexibility. This is a consideration to be taken into account for further studies on the mouthpiece design and for personalization attempts.

Changes in window and table lengths do not present significant effects on any of the features. Both parameters may affect the reed oscillation and hence are commonly considered to affect timbre [12]. According to the results, it is possible to conclude that they do not have any significant influence on brightness. However, they may still affect other timbral characteristics such as edge or projection.

The analysis of the results also reveals considerably nonlinear feature-parameter relations. The most notable ones are loudness- $h_{\text {tip }}$, brightness- $h_{\text {baffle }}$ and brightness- $s_{\text {throat }}$. The results regarding tip opening $\left(h_{\text {tip }}\right)$ may be because of the fixed reed strength, as stated in Section 5. Baffle and throat may have some interactions, since both of them show unexpected behavior for brightness between levels 1 and 2 .

These experimental findings were then evaluated via a user study with five experienced saxophonists. The results of the user study show that participants were aware of changes in mouthpiece features and, in most cases, were able to judge their changes demonstrated via benchmark mouthpieces during the sessions. Besides, they were also satisfied with the mouthpieces personalized in the proposed way. However, they also provided key insights pointing out room 
for improvement. As they stated, configuring a mouthpiece based on their current ones might make the process more easily graspable. Likewise, comments on the tip opening and lay length indicate that user habits should be more carefully considered in devising a personalization model.

\section{Conclusion}

This study has investigated how design changes in the mouthpiece influence the acoustical and playability features of saxophone mouthpieces. For this purpose, four mouthpiece features related to four quantitative measures were chosen and the variations in these were observed according to the changes in nine design parameters. The evaluation of the mouthpieces with an artificial blowing machine (for resistance, loudness and brightness) and via a blind test (for flexibility) quantified to which extent each design parameter affects each mouthpiece feature. These objective results were then interpreted in a mouthpiece personalization model, where the identified parameters are tuned to tailor the mouthpiece features. Finally, a user study was devised to verify whether the players could recognize the changes in the mouthpiece when asked about each feature. According to the results, users judged the feature changes correctly 7 out of 10 times and they preferred the personalized mouthpieces 4 out of 5 times.

The exact quantities of parameter effects are naturally valid only in the proposed mouthpiece model, but the relative effects would be still valid in other mouthpiece designs. The experiment used a fractional factorial design, where parameter interactions are not explicitly considered. Therefore, in future studies, insignificant parameters and levels may be eliminated, and hence an experiment with a full factorial design would be more feasible and provide more robust results. In terms of mouthpiece playability, all the major aspects are covered in the study. Brightness is the only timbre attribute considered, as it is arguably the most significant for the players, and it has a quantitative measure. Future studies may also investigate other timbral characteristics, as long as objective measures for those are introduced. Key insights from the user study indicated that players prefer direct control over facing parameters (lay length and tip opening) and they are more comfortable stating their preferences with respect to their own mouthpieces. A library and a classification of the most common mouthpiece designs may provide a more satisfactory and accurate personalization experience. Future personalizing approaches may adopt these principles.

The findings of this study provide a critical contribution to the understanding of saxophone mouthpiece design. Identified parameter-feature relationships are both quantitative and free of player subjectivity. This study showed the entangled relationships among design parameters affecting the mouthpiece-reed-player interaction, a fact that supports the need for more research in the field. The description of these relationships provides useful insights for new mouthpiece designs or for personalization cases to deliver the performance requirements in a fine-tuned manner.

\section{Conflict of interest}

Author declared no conflict of interests.

\section{Acknowledgments}

This research was supported by FARO S.p.A. (Ornago, Italy) and by the Austrian Science Fund (FWF): P28655N32. The authors would like to thank Hans de Jong, professor of classical saxophone at the Royal Conservatoire Antwerp, as well as the saxophone students who participated in the user study.

\section{References}

1. N.H. Fletcher, T. Rossing: The physics of musical instruments. Springer Science \& Business Media, 1998.

2. J.-M. Chen, J. Smith, J. Wolfe: Saxophone acoustics: Introducing a compendium of impedance and sound spectra. Acoustics Australia 37, 1-19 (2009), 18-23.

3. J. Kergomard, P. Guillemain, P. Sanchez, C. Vergez, J.-P. Dalmont, B. Gazengel, S. Karkar: Role of the resonator geometry on the pressure spectrum of reed conical instruments. Acta Acustica United with Acustica 105, 2 (2019) 368-380. https://doi.org/10.3813/AAA.919320.

4. L. Teal: The Art of Saxophone Playing. The Art of Series. Summy-Birchard, 1963.

5. V.R. Hasbrook: Alto Saxophone Mouthpiece Pitch and Its Relation to Jazz and Classical Tone Qualities. University of Illinois at Urbana-Champaign, 2005.

6. J.-P. Dalmont, C. Frappe: Oscillation and extinction thresholds of the clarinet: Comparison of analytical results and experiments. The Journal of the Acoustical Society of America 122, 2 (2007) 1173-1179. https://doi.org/10.1121/1.2747197.

7. V. Chatziioannou, A. Hofmann, M. Pàmies-Vilà: An artificial blowing machine to investigate single-reed woodwind instruments under controlled articulation conditions, in Proceedings of Meetings on Acoustics 174ASA, 4-8 December 2017, New Orleans, Vol. 31. 2017, 035003 p. https://doi.org/10.1121/2.0000794.

8. J.-P. Dalmont, J. Gilbert, S. Ollivier: Nonlinear characteristics of single-reed instruments: Quasistatic volume flow and reed opening measurements. The Journal of the Acoustical Society of America 114, 4 (2003) 2253-2262. https://asa. scitation.org/doi/10.1121/1.1603235.

9.F. Avanzini, M. Van Walstijn: Modelling the mechanical response of the reed-mouthpiece-lip system of a clarinet. Part I. A one-dimensional distributed model. Acta Acustica United with Acustica 90, 3 (2004) 537-547.

10. V. Chatzionnou: Forward and inverse modelling of singlereed woodwind instruments with application to digital sound synthesis. PhD thesis.Queen's University Belfast, 2011.

11.F.S. Wyman: An Acoustical Study of Alto Saxophone Mouthpiece Chamber Design. Eastman School of Music, University of Rochester, 1972.

12. F. Celentano, R. DiPasquale, E. Simoneau, N. May, Z. Shahbazi, S. Shahbazmohamadi: Reverse engineering and geometric optimization for resurrecting antique saxophone sound using micro-ct and additive manufacturing. Journal of Computing and Information Science in Engineering 17 (2017). https://doi.org/10.1115/1.4037180.

13. M. Ozdemir, G. Cascini: An experiment-driven masspersonalisation model: Application to saxophone mouthpiece production, in Proceedings of the Design Society: DESIGN Conference, Vol. 1. 2020, pp. 1037-1046. https://doi.org/ 10.1017/dsd.2020.169. 
14. G. Taguchi, S. Chowdhury, Y. Wu: Taguchi's Quality Engineering Handbook. John Wiley \& Sons, Inc., 2004.

15. M.R. Pipes: A comparison of saxophone mouthpieces using fourier analysis to quantify perceived timbre. $\mathrm{PhD}$ thesis, University of Northern Colorado, 2018.

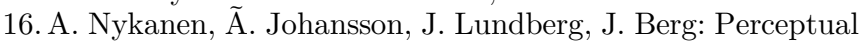
and acoustical dimensions of saxophone sound, in Proceedings of Forum Acusticum 2005, 29 August-2 September 2005, Budapest. 2005.

17. A. Nykanen, A. Johansson, J. Lundberg, J. Berg: Modelling perceptual dimensions of saxophone sounds. Acta Acustica United with Acustica 95 (2009) 539-549.

18. D.L. Wessel: Timbre space as a musical control structure. Computer Music Journal 3 (1979) 45-52.

19. R.A. Smith, D.M.A. Mercer: Possible causes of woodwind tone colour. Journal of Sound and Vibration 32, 3 (1974) 347 - IN1. https://doi.org/10.1016/S0022-460X(74)80090-8.

20. H. Pinksterboer: The Rough Guide to Saxophone. Rough Guide Tipbooks Series. Rough Guides, 2000.

21. D. Liebman: Developing a Personal Saxophone Sound. Dorn Publications, 1989.

22. V. Lorenzoni, D. Ragni: Experimental investigation of the flow inside a saxophone mouthpiece by particle image velocimetry. The Journal of the Acoustical Society of America 131, 1 (2012) 715-721. https://doi.org/10.1121/1.3651795.

23. A. Damodaran, M. Sugavaneswaran, L. Lessard: An overview of additive manufacturing technologies for musical wind instruments. SN Applied Sciences 3 (2021) 162. https://doi. org/10.1007/s42452-021-04170-x.

24. A. Bacciaglia, A. Ceruti, A. Liverani: Evaluation of 3D printed mouthpieces for musical instruments. Rapid Prototyping Journal 26 (2019) 577-584. https://doi.org/10.1108/ RPJ-07-2019-0187.

25. B. Hang, G. Stetten: Novel saxophone mouthpiece design through additive manufacturing. University of Pittsburgh, 2017.

26. P. Eveno, M. Carron: How is a custom saxophone mouthpiece even possible? https://www.syos.co/en/blog/acoustics/how-is-saxophone-mouthpiece-customization-possible. Accessed: 2021-03-05.

27. V. Lorenzoni, Z. Doubrovski, J. Verlinden: Embracing the digital in instrument making: Towards a musician-tailored mouthpiece by 3D printing, in Proceedings of Stockholm Music Acoustics Conference, 30 July-3 August 2013, Stockholm. 2013, pp. 419-424.

28. M. Carron, T. Rotureau, F. Dubois, N. Misdariis, P. Susini: Speaking about sounds: a tool for communication on sound features. Journal of Design Research 15, 2 (2017) 85-109. https://doi.org/10.1504/JDR.2017.086749.

29. J.M. Chen, J. Smith, J. Wolfe: Experienced saxophonists learn to tune their vocal tracts. Science 319, 5864 (2008) 776. https://doi.org/10.1126/science.1151411.

30. J. Wolfe, N.H. Fletcher, J. Smith: The interactions between wind instruments and their players. Acta Acustica United with Acustica 101, 2 (2015) 211-223. https://doi.org/ 10.3813/AAA.918820.

31. G.P. Scavone, A. Lefebvre, A.R. da Silva: Measurement of vocal-tract influence during saxophone performance. The Journal of the Acoustical Society of America 123, 4 (2008) 2391-2400. https://doi.org/10.1121/1.2839900.

32. M.M. Tseng, R.J. Jiao, C. Wang; Design for mass personalization. CIRP Annals 59, 1 (2010) 175-178. https://doi. org/10.1016/j.cirp.2010.03.097.

33. M. Pàmies-Vilà, A. Hofmann, V. Chatziioannou: Analysis of tonguing and blowing actions during clarinet performance. Frontiers in Psychology 9 (2018) 617. https://doi.org/ 10.3389/fpsyg.2018.00617.
34. D.E. Norris: Measuring pitch flexibility on the saxophone. PhD thesis, Kansas State University, 1974.

35. T. Liley, T. Dryer-Beers, K. Horch, D. Roach, N. Turner, S. Trier, G. Lewin, C. Davis: The Cambridge companion to the saxophone. Cambridge University Press, 1998. https://doi. org/10.1017/CCOL9780521593489.

36. Mouthpieces - YANAGISAWA Saxophones Official website. http://www.yanagisawasax.co.jp/en/saxophones/view/529. Accessed: 2021-05-20.

37. V. Chatziioannou, S. Schmutzhard, M. Pàmies-Vilà, A. Hofmann: Investigating clarinet articulation using a physical model and an artificial blowing machine. Acta Acustica United with Acustica 105, 4 (2019) 682-694. https://doi.org/ 10.1121/2.0000794.

38. A. Almeida, D. George, J. Smith, J. Wolfe: The clarinet: How blowing pressure, lip force, lip position and reed "hardness" affect pitch, sound level, and spectrum. The Journal of the Acoustical Society of America 134, 3 (2013) 2247-2255. https://doi.org/10.1121/1.4816538.

39. M. Pàmies-Vilà: Expressive performance on single-reed woodwind instruments: An experimental characterisation of articulatory actions. $\mathrm{PhD}$ thesis, University of Music and Performing Arts Vienna, 2021. https://www.doi.org/ 10.21939/exper-pamvil.

40. S. McAdams, K. Siedenburg, Perception and cognition of musical timbre, in Foundations in music psychology: Theory and research, The MIT Press. 2019, pp. 71-120.

41. G. Peeters, B.L. Giordano, P. Susini, N. Misdariis, S. McAdams: The timbre toolbox: Extracting audio descriptors from musical signals. The Journal of the Acoustical Society of America 130, 5 (2011) 2902-2916. https://doi.org/10.1121/ 1.3642604 .

42. B. Gazengel, J.P. Dalmont, J.F. Petiot: Link between objective and subjective characterizations of $\mathrm{Bb}$ clarinet reeds. Applied Acoustics 106 (2016) 155-166. https://doi. org/10.1016/j.apacoust.2015.12.015.

43. F.E. Harrell: Regression Modeling Strategies, Vol. 45 of Springer Series in Statistics. Springer International Publishing, Cham, 2015.

44. J.-P. Dalmont, J. Gilbert, J. Kergomard, S. Ollivier: An analytical prediction of the oscillation and extinction thresholds of a clarinet. The Journal of the Acoustical Society of America 118, 5 (2005) 3294-3305.

45. S. Wang, E. Maestre, G. Scavone: Acoustical modeling of the saxophone mouthpiece as a transfer matrix. The Journal of the Acoustical Society of America 149, 3 (2021) 1901-1912. https://doi.org/10.1121/10.0003814.

46. B. Andrieux, V. Gibiat, J. Selmer: Modeling of a woodwind mouthpiece using a finite-element method and characterization of its acoustic input impedance, in Proceedings of the International Symposium on Music Acoustics (ISMA) 2014, 7-12 July 2014, Le Mans. 2014, pp. 7-12.

47. B.B. McShane, D. Gal: Statistical significance and the dichotomization of evidence. Journal of the American Statistical Association 112, 519 (2017) 885-895. https://doi.org/ 10.1080/01621459.2017.1289846.

48. B.B. McShane, D. Gal, A. Gelman, C. Robert, J.L. Tackett: Abandon statistical significance. The American Statistician 73, Suppl. 1 (2019) 235-245. https://doi.org/10.1080/ 00031305.2018.1527253.

49. M. Carron: The baffle: A true game changer. https://www. syos.co/blog/gear/baffle-saxophone-mouthpiece. Accessed: 2021-03-05.

50. M. Pàmies-Vilà, A. Hofmann, V. Chatziioannou: The influence of the vocal tract on the attack transients in clarinet playing. Journal of New Music Research 49, 2 (2020) 126-135. https://doi.org/10.1080/09298215.2019.1708412. 


\section{Appendix}

\section{Additional material}

Table A.1. Experimental layout using L27 orthogonal arrays.

\begin{tabular}{|c|c|c|c|c|c|c|c|c|c|}
\hline & & & & Param & eter level & & & & \\
\hline & $s_{\text {chamber }}$ & $h_{\text {baffle }}$ & $s_{\text {throat }}$ & $r_{\text {throat }}$ & $r_{\text {chamber }}$ & $l_{\text {window }}$ & $l_{\text {table }}$ & $l_{\text {lay }}$ & $\overline{h_{\text {tip }}}$ \\
\hline 1 & 1 & 1 & 1 & 1 & 1 & 1 & 1 & 1 & 1 \\
\hline 2 & 1 & 1 & 1 & 1 & 2 & 2 & 2 & 2 & 2 \\
\hline 3 & 1 & 1 & 1 & 1 & 3 & 3 & 3 & 3 & 3 \\
\hline 4 & 1 & 2 & 2 & 2 & 1 & 1 & 1 & 2 & 2 \\
\hline 5 & 1 & 2 & 2 & 2 & 2 & 2 & 2 & 3 & 3 \\
\hline 6 & 1 & 2 & 2 & 2 & 3 & 3 & 3 & 1 & 1 \\
\hline 7 & 1 & 3 & 3 & 3 & 1 & 1 & 1 & 3 & 3 \\
\hline 8 & 1 & 3 & 3 & 3 & 2 & 2 & 2 & 1 & 1 \\
\hline 9 & 1 & 3 & 3 & 3 & 3 & 3 & 3 & 2 & 2 \\
\hline 10 & 2 & 1 & 2 & 3 & 1 & 2 & 3 & 1 & 2 \\
\hline 11 & 2 & 1 & 2 & 3 & 2 & 3 & 1 & 2 & 3 \\
\hline 12 & 2 & 1 & 2 & 3 & 3 & 1 & 2 & 3 & 1 \\
\hline 13 & 2 & 2 & 3 & 1 & 1 & 2 & 3 & 2 & 3 \\
\hline 14 & 2 & 2 & 3 & 1 & 2 & 3 & 1 & 3 & 1 \\
\hline 15 & 2 & 2 & 3 & 1 & 3 & 1 & 2 & 1 & 2 \\
\hline 16 & 2 & 3 & 1 & 2 & 1 & 2 & 3 & 3 & 1 \\
\hline 17 & 2 & 3 & 1 & 2 & 2 & 3 & 1 & 1 & 2 \\
\hline 18 & 2 & 3 & 1 & 2 & 3 & 1 & 2 & 2 & 3 \\
\hline 19 & 3 & 1 & 3 & 2 & 1 & 3 & 2 & 1 & 3 \\
\hline 20 & 3 & 1 & 3 & 2 & 2 & 1 & 3 & 2 & 1 \\
\hline 21 & 3 & 1 & 3 & 2 & 3 & 2 & 1 & 3 & 2 \\
\hline 22 & 3 & 2 & 1 & 3 & 1 & 3 & 2 & 2 & 1 \\
\hline 23 & 3 & 2 & 1 & 3 & 2 & 1 & 3 & 3 & 2 \\
\hline 24 & 3 & 2 & 1 & 3 & 3 & 2 & 1 & 1 & 3 \\
\hline 25 & 3 & 3 & 2 & 1 & 1 & 3 & 2 & 3 & 2 \\
\hline 26 & 3 & 3 & 2 & 1 & 2 & 1 & 3 & 1 & 3 \\
\hline 27 & 3 & 3 & 2 & 1 & 3 & 2 & 1 & 2 & 1 \\
\hline
\end{tabular}

Table A.2. Analysis of variance for oscillation threshold, $R^{2}=94.69 \%$.

\begin{tabular}{lcccc}
\hline Source & $d f$ & Seq SS & $F$ & $p$ \\
\hline$h_{\text {tip }}$ & 2 & 35.2844 & 33.15 & 0 \\
$l_{\text {lay }}$ & 2 & 27.907 & 26.22 & 0 \\
$s_{\text {chamber }}$ & 2 & 4.0223 & 3.78 & 0.070 \\
$r_{\text {chamber }}$ & 2 & 3.2857 & 3.09 & 0.101 \\
$h_{\text {baffle }}$ & 2 & 3.1702 & 2.98 & 0.108 \\
$l_{\text {table }}$ & 2 & 1.2113 & 1.14 & 0.367 \\
$s_{\text {throat }}$ & 2 & 0.3686 & 0.35 & 0.717 \\
$l_{\text {window }}$ & 2 & 0.3428 & 0.32 & 0.734 \\
$r_{\text {throat }}$ & 2 & 0.2896 & 0.27 & 0.769 \\
Residual error & 8 & 4.2579 & & \\
Total & 26 & 80.1397 & & \\
\hline
\end{tabular}

Table A.3. Analysis of variance for oscillation threshold after model reduction, $R^{2}=91.93 \%$.

\begin{tabular}{lcccc}
\hline Source & $d f$ & Seq SS & $F$ & $p$ \\
\hline$h_{\text {tip }}$ & 2 & 35.284 & 43.63 & 0 \\
$l_{\text {lay }}$ & 2 & 27.907 & 34.51 & 0 \\
$s_{\text {chamber }}$ & 2 & 4.022 & 4.97 & 0.021 \\
$r_{\text {chamber }}$ & 2 & 3.286 & 4.06 & 0.037 \\
$h_{\text {baffle }}$ & 2 & 3.17 & 3.92 & 0.041 \\
Residual error & 16 & 6.47 & & \\
Total & 26 & 80.14 & & \\
\hline
\end{tabular}

Table A.4. Analysis of variance for external sound level, $R^{2}=83.93 \%$.

\begin{tabular}{lcccc}
\hline Source & $d f$ & Seq SS & $F$ & $p$ \\
\hline$s_{\text {throat }}$ & 2 & 0.054683 & 6.76 & 0.019 \\
$h_{\text {tip }}$ & 2 & 0.036611 & 4.53 & 0.048 \\
$s_{\text {chamber }}$ & 2 & 0.022128 & 2.74 & 0.124 \\
$l_{\text {lay }}$ & 2 & 0.017281 & 2.14 & 0.180 \\
$r_{\text {throat }}$ & 2 & 0.015429 & 1.91 & 0.210 \\
$r_{\text {chamber }}$ & 2 & 0.011408 & 1.41 & 0.299 \\
$h_{\text {baffle }}$ & 2 & 0.009452 & 1.17 & 0.359 \\
$l_{\text {table }}$ & 2 & 0.001781 & 0.22 & 0.807 \\
$l_{\text {window }}$ & 2 & 0.000196 & 0.02 & 0.976 \\
Residual error & 8 & 0.032342 & & \\
Total & 26 & 0.201312 & & \\
\hline
\end{tabular}

Table A.5. Analysis of variance for external sound level after model reduction, $R^{2}=72.59 \%$.

\begin{tabular}{lcccc}
\hline Source & $d f$ & Seq SS & $F$ & $p$ \\
\hline$s_{\text {throat }}$ & 2 & 0.05468 & 7.93 & 0.004 \\
$h_{\text {tip }}$ & 2 & 0.03661 & 5.31 & 0.017 \\
$s_{\text {chamber }}$ & 2 & 0.02213 & 3.21 & 0.067 \\
$l_{\text {lay }}$ & 2 & 0.01728 & 2.51 & 0.113 \\
$r_{\text {throat }}$ & 2 & 0.01543 & 2.24 & 0.139 \\
Residual error & 16 & 0.05518 & & \\
Total & 26 & 0.20131 & & \\
\hline
\end{tabular}


Table A.6. Analysis of variance for spectral centroid, $R^{2}=86.17 \%$.

\begin{tabular}{lcccc}
\hline Source & $d f$ & Seq SS & $F$ & $p$ \\
\hline$l_{\text {lay }}$ & 2 & 3654293 & 6.93 & 0.018 \\
$s_{\text {throat }}$ & 2 & 3571621 & 6.78 & 0.019 \\
$h_{\text {baffle }}$ & 2 & 2035572 & 3.86 & 0.067 \\
$l_{\text {table }}$ & 2 & 1623841 & 3.08 & 0.102 \\
$h_{\text {tip }}$ & 2 & 842065 & 1.60 & 0.261 \\
$s_{\text {chamber }}$ & 2 & 767167 & 1.46 & 0.289 \\
$r_{\text {throat }}$ & 2 & 337242 & 0.64 & 0.552 \\
$r_{\text {chamber }}$ & 2 & 300289 & 0.57 & 0.587 \\
$l_{\text {window }}$ & 2 & 4949 & 0.01 & 0.991 \\
Residual error & 8 & 2108169 & & \\
Total & 26 & 15245208 & & \\
\hline
\end{tabular}

Table A.7. Analysis of variance for spectral centroid after model reduction, $R^{2}=60.75 \%$.

\begin{tabular}{lcccc}
\hline Source & $d f$ & Seq SS & $F$ & $p$ \\
\hline$l_{\text {lay }}$ & 2 & 3654293 & 6.11 & 0.009 \\
$s_{\text {throat }}$ & 2 & 3571621 & 5.97 & 0.009 \\
$h_{\text {baffle }}$ & 2 & 2035572 & 3.40 & 0.053 \\
Residual error & 22 & 5983722 & & \\
Total & 26 & 15245208 & & \\
\hline
\end{tabular}

Table A.8. Analysis of variance for pitch interval, $R^{2}=83 \%$.

\begin{tabular}{lcccc}
\hline Source & $d f$ & Seq SS & $F$ & $p$ \\
\hline$h_{\text {baffle }}$ & 2 & 5033.9 & 7.68 & 0.014 \\
$s_{\text {chamber }}$ & 2 & 3176.5 & 4.85 & 0.042 \\
$h_{\text {tip }}$ & 2 & 1897.2 & 2.90 & 0.113 \\
$r_{\text {throat }}$ & 2 & 812.1 & 1.24 & 0.34 \\
$r_{\text {chamber }}$ & 2 & 718.7 & 1.10 & 0.379 \\
$l_{\text {table }}$ & 2 & 500.1 & 0.76 & 0.497 \\
$s_{\text {throat }}$ & 2 & 296.1 & 0.45 & 0.652 \\
$l_{\text {lay }}$ & 2 & 190.7 & 0.29 & 0.755 \\
$l_{\text {window }}$ & 2 & 174.5 & 0.27 & 0.773 \\
Residual error & 8 & 2620.7 & & \\
Total & 26 & 15420.5 & & \\
\hline
\end{tabular}

Table A.9. Analysis of variance for pitch interval after model reduction, $R^{2}=65.55 \%$.

\begin{tabular}{lcccc}
\hline Source & $d f$ & Seq SS & $F$ & $p$ \\
\hline$h_{\text {baffle }}$ & 2 & 5034 & 9.47 & 0.001 \\
$s_{\text {chamber }}$ & 2 & 3177 & 5.98 & 0.009 \\
$h_{\text {tip }}$ & 2 & 1897 & 3.57 & 0.047 \\
Residual error & 20 & 5313 & & \\
Total & 26 & 15421 & & \\
\hline
\end{tabular}



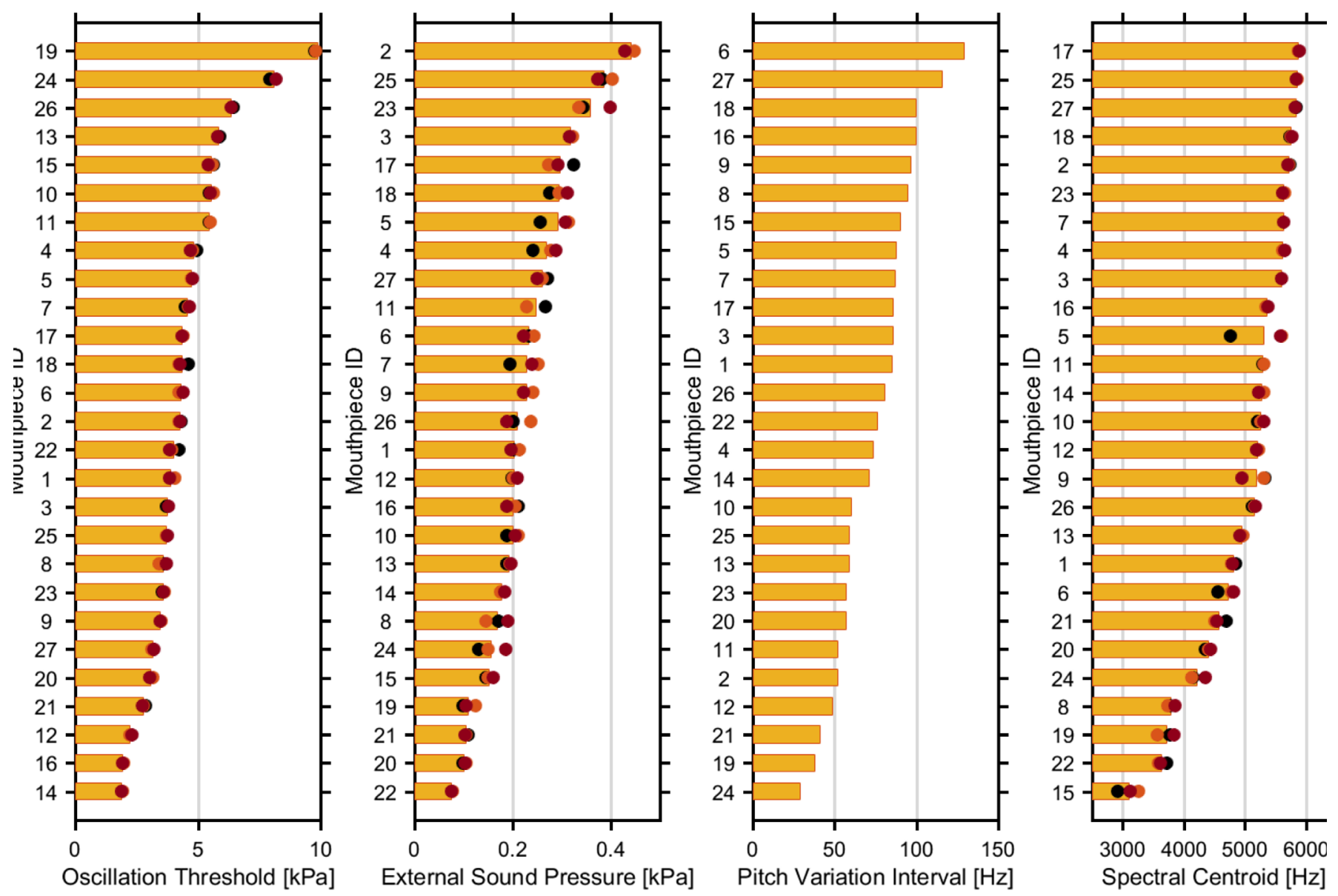

Figure A.1. Mean values for the four tested features on each mouthpiece (bars), with indication of the three values of the repeated tests (dots). The standard deviations of the three measurements averaged along all mouthpieces are: $0.08 \mathrm{kPa}$ for Oscillation threshold, $0.015 \mathrm{kPa}$ for External sound, $68.9 \mathrm{~Hz}$ for Spectral centroid. Mouthpieces are ordered by descending feature values.

Cite this article as: Ozdemir M, Chatziioannou V, Verlinden J, Cascini G, \& Pàmies-Vilà M. 2021. Towards 3D printed saxophone mouthpiece personalization: Acoustical analysis of design variations. Acta Acustica, 5, 46. 